MATHEMATICS OF COMPUTATION

Volume 67, Number 222, April 1998, Pages 553-591

S 0025-5718(98)00937-5

\title{
A CONVERGENCE THEOREM FOR THE FAST MULTIPOLE METHOD FOR 2 DIMENSIONAL SCATTERING PROBLEMS
}

\author{
CHRISTOPHE LABREUCHE
}

\begin{abstract}
The Fast Multipole Method (FMM) designed by V. Rokhlin rapidly computes the field scattered from an obstacle. This computation consists of solving an integral equation on the boundary of the obstacle. The main result of this paper shows the convergence of the FMM for the two dimensional Helmholtz equation. Before giving the theorem, we give an overview of the main ideas of the FMM. This is done following the papers of V. Rokhlin. Nevertheless, the way we present the FMM is slightly different. The FMM is finally applied to an acoustic problem with an impedance boundary condition. The moment method is used to discretize this continuous problem.
\end{abstract}

\section{INTRODUCTION}

Many different numerical methods are available for the resolution of acoustic or electromagnetic equations outside a bounded obstacle. The first class of numerical methods can be called "volume methods" [10]. The open space outside the obstacle is bounded by an artificial surface. The domain inside the artificial surface is meshed and a proper boundary condition on the artificial surface must be introduced. The main drawback of this approach is that it leads to a huge number of unknowns. If the obstacle is impenetrable, an alternative method can be used based on integral equations over the boundary of the obstacle [5]. The number of unknowns $n$ arising when discretizing such equations is relatively small, since only the boundary of the obstacle is meshed. But, in return, since the kernels of the integral operators are non-local, the discretization leads to a dense matrix $\mathbf{Z}$. When the wave number $k$ times the size of the obstacle is large, the number of unknowns $n$ necessary to get good accuracy can be very large. For such large scale problems, the inversion of the dense matrix $\mathbf{Z}$ is very tedious. Inverting a dense $n \times n$ matrix with a direct solver, such as the LU decomposition, is an order $n^{3}$ procedure. On the other hand, the inversion can be performed by the mean of an iterative method, such as a conjugate gradient type algorithm. Each iteration typically requires the computation of a couple of scalar products and at least one multiplication of a vector by the matrix $\mathbf{Z}$. The number of operations for this is proportional to $n^{2}$, since the matrix $\mathbf{Z}$ is dense. Thus the overall cost of an iterative method is proportional to $n^{2}$ times the number of iterations.

Received by the editor December 11, 1995 and, in revised form, October 7, 1996.

1991 Mathematics Subject Classification. Primary 41A58, 35J05, 65N30.

Key words and phrases. Fast Multipole Method, Helmholtz equation, Hankel function. 
Using second kind integral equations and an appropriate preconditioner, the number of iterations can be controlled. But the major task in order to get an efficient acoustic or electromagnetic solver consists in writing a fast matrix-vector multiplication routine. Methods that perform the matrix-vector multiplication in an order $n^{r}$ operations, with $r$ significantly lower than 2, are called "fast methods". In the literature, three main methods are designed to be fast in the previous sense. The Fast Multipole Method (FMM) [14, 15, 16, 17, 18], which is the main focus of this article, is the most popular one. The two others are the wavelet-based method $[8,9,4]$ and the Impedance Matrix Localization method $[2,3]$. The idea of these two latter methods is similar and has nothing to do with the FMM: The dense matrix $\mathbf{Z}$ is approximated by a sparse matrix after an appropriate change of basis elements.

The main reference to the FMM in two dimension is the original paper of V. Rokhlin [14]. The convergence analysis of this method does not seem to appear in the literature so far. This has actually been the main criticism of this method. The purpose of this paper is to provide a rigorous proof for the acoustic problem (Helmholtz equation) in two dimension. The proof is quite long and technical. This emphasizes the subtleties and the complexity of the FMM.

In fact, there exists several FMM corresponding to different physical problems. For instance, there is one FMM designed for the computation of long-range interactions in particle systems (molecular dynamics simulations), which is described in [6]. A proof of the convergence of the FMM in this case has been done by $\mathrm{H}$. Petersen et al. [12]. But this work cannot be used for the Helmholtz equation.

The FMM presented here is the basic version, leading to a matrix-vector multiplication in $O\left(n^{3 / 2}\right)$ operations. We would like to point out that more elaborate versions of the FMM reduce the cost to $O\left(n^{4 / 3}\right)$ [14], or even $O(n \log n)$ [13] in its multistep version.

To be consistent with the convergence proof given here, the FMM is presented in its mathematical form. Most articles on this method insist on the physical basis of this method which is the well-known "multipole method" designed to solve multiple obstacle problems [11]. We rather dwell on the mathematical trickery of the FMM which is the matrix-vector multiplication. Accordingly, the main feature of the FMM is a special approximation of the fundamental solution to the Helmholtz equation. The rest of the FMM is quite straightforward.

The layout of this paper is as follows. In Section 2, the necessary notation is introduced and we give the main ideas of the FMM. In Section 3, the approximate form of the fundamental solution to the Helmholtz equation is derived. But the convergence analysis and the justification of all the calculations done in Section 3, is performed in Section 4. In this approximate form, an integral appears. Numerically, this integral must be discretized. The convergence analysis of the discretized approximate form is thoroughly investigated in Section 5. In Section 6, the convergence of the derivatives of the kernel is proved, and then we show that the approximate matrix (defined with the approximate kernel) converges to the exact matrix Z. Finally, the fast matrix-vector multiplication is explained in Section 7 . Three appendices can be found at the end of the paper (respectively Sections 9, 10 and 11). In Section 9, some formulae on Bessel and Hankel functions are recalled. The basic form of the multipole formula is given in Section 10. The last section gives some further results which are very useful in the convergence analysis. The 
Appendix to this paper contains equations (35) through (47), and Propositions 8 through 14.

\section{MAIN IDEAS OF THE FMM}

Let us begin with some notation: $\mathbb{N}^{*}$ is the set of all strictly positive integers, $\mathbb{N}$ is the set of all non-negative integers, $\mathbb{Z}$ is the set of all integers, $\mathbb{R}$ is the set of all real numbers, and $\mathbb{C}$ is the set of all complex numbers. Throughout this paper, matrices, vectors and points will be typed in bold characters, whereas scalar numbers will be typed with standard letters. If $\mathbf{x}$ and $\mathbf{y}$ are two points of the plane $\mathbb{R}^{2}$, the vector $\mathbf{x}-\mathbf{y}$ is defined by its norm $|\mathbf{x}-\mathbf{y}|$ and by $\arg (\mathbf{x}-\mathbf{y})$ which represents the angle between the horizontal axis and the vector $\mathbf{x}-\mathbf{y}$. The scalar product between two vectors $\mathbf{u}$ and $\mathbf{v}$ of $\mathbb{R}^{2}$ is denoted by $\mathbf{u} \cdot \mathbf{v}$. The purely imaginary number $\sqrt{-1}$ is denoted by $\imath$. The empty set is denoted by $\emptyset$. The wave number denoted by $k$ will be assumed to belong to the complex upper half plane $\Im(k) \geq 0$. Let us define now the Bessel and the Hankel functions [1]: $J_{m}(z)$ is the Bessel function of order $m$ and $\operatorname{argument} z ; H_{m}^{(1)}(z)$ is the Hankel function of the first kind and order $m$; and $K_{m}(z):=\frac{\imath \pi}{2}(\imath)^{m} H_{m}^{(1)}(\imath z)$ is the modified Hankel function of order $m$.

Let us consider an open bounded domain $\Omega_{i}$ of $\mathbb{R}^{2}$ and denote its complement by $\Omega:=\mathbb{R}^{2} \backslash \bar{\Omega}_{i}$. $\Gamma$ is the boundary of $\Omega_{i}$. We denote by $\mathbf{n}$ the outer unit normal of $\partial \Omega_{i}$, that is the normal going from $\Omega_{i}$ to $\Omega$. Let us introduce the following system

$$
\left\{\begin{array}{l}
\Delta u+k^{2} u=0 \text { in } \Omega, \\
\frac{\partial u}{\partial \mathbf{n}}+\imath \zeta u=g \text { on } \Gamma, \\
\lim _{|\mathbf{x}| \rightarrow \infty} \sqrt{|\mathbf{x}|}\left(\frac{\partial}{\partial|\mathbf{x}|} u(\mathbf{x})-\imath k u(\mathbf{x})\right)=0 .
\end{array}\right.
$$

The wave number is assumed to satisfy $\Im(k) \geq 0$, so that the outgoing Sommerfeld condition makes sense. The boundary condition considered here is the impedance one (characterized by the impedance function $\zeta$ depending on $k \in \mathbb{C}$ and $\mathbf{x} \in \Gamma$ ). The analysis below can be extended with no difficulty to any classical boundary condition. With some assumptions on $\zeta$ (namely, $\Re(k \bar{\zeta}) \geq 0$ for all $k$ such that $\Im(k) \geq 0)$ the above exterior problem has a unique solution ([5]). Moreover, $u$ can be given as the solution of an integral equation over the boundary $\Gamma$. The field $u$ can be written as a combination of single layer and the double layer potentials

$$
u(\mathbf{x})=-\imath \tilde{V}(\zeta v)(\mathbf{x})-\tilde{K}(v)(\mathbf{x}), \text { for } \mathbf{x} \in \Omega,
$$

where the potential $v$ defined on $\Gamma$ is to be determined. The operators

$$
\tilde{V}(v)(\mathbf{x})=\int_{\Gamma} G\left(\mathbf{x}, \mathbf{x}^{\prime}\right) v\left(\mathbf{x}^{\prime}\right) d \gamma\left(\mathbf{x}^{\prime}\right), \quad \tilde{K}(v)(\mathbf{x})=\int_{\Gamma} \frac{\partial G\left(\mathbf{x}, \mathbf{x}^{\prime}\right)}{\partial \mathbf{n}\left(\mathbf{x}^{\prime}\right)} v\left(\mathbf{x}^{\prime}\right) d \gamma\left(\mathbf{x}^{\prime}\right)
$$

are defined for $\mathbf{x} \notin \Gamma$. The fundamental solution to the Helmholtz equation is $G\left(\mathbf{x}, \mathbf{x}^{\prime}\right)=\frac{\imath}{4} H_{0}^{(1)}\left(k\left|\mathbf{x}-\mathbf{x}^{\prime}\right|\right)$. The advantage of integral representations is that the Helmholtz equation and the outgoing Sommerfeld condition are automatically satisfied. Then the potential $v$ is determined by requiring that the impedance boundary condition is satisfied, leading to [5]

$$
D(v)+\imath\left(\zeta K(v)+K^{t}(\zeta v)\right)-\zeta V(\zeta v)=-g
$$


where the singular operators

$$
\begin{aligned}
V(w)(\mathbf{x}) & =\int_{\Gamma} G\left(\mathbf{x}, \mathbf{x}^{\prime}\right) w\left(\mathbf{x}^{\prime}\right) d \gamma\left(\mathbf{x}^{\prime}\right) \\
K(v)(\mathbf{x}) & =\int_{\Gamma} \frac{\partial G\left(\mathbf{x}, \mathbf{x}^{\prime}\right)}{\partial \mathbf{n}\left(\mathbf{x}^{\prime}\right)} v\left(\mathbf{x}^{\prime}\right) d \gamma\left(\mathbf{x}^{\prime}\right) \\
K^{t}(w)(\mathbf{x}) & =\int_{\Gamma} \frac{\partial G\left(\mathbf{x}, \mathbf{x}^{\prime}\right)}{\partial \mathbf{n}(\mathbf{x})} w\left(\mathbf{x}^{\prime}\right) d \gamma\left(\mathbf{x}^{\prime}\right), \\
D(v)(\mathbf{x}) & =\int_{\Gamma} \frac{\partial^{2} G\left(\mathbf{x}, \mathbf{x}^{\prime}\right)}{\partial \mathbf{n}(\mathbf{x}) \partial \mathbf{n}\left(\mathbf{x}^{\prime}\right)} v\left(\mathbf{x}^{\prime}\right) d \gamma\left(\mathbf{x}^{\prime}\right)
\end{aligned}
$$

are defined for $\mathbf{x} \in \Gamma$. Notice that (2) is only one possible representation of $u$. The reason for taking (2), is that the four singular operators $V, K, K^{t}$ and $D$ are present in the integral equation (3). Hence the convergence analysis for all four integral operators will be performed in this paper.

The pseudo-differential operator $D$ has a hypersingular kernel and thus must be viewed in the sense of finite-parts ([7]). The discretization of (3) with any numerical method (moment method, collocation, finite differences) leads to a dense matrix $\mathbf{Z}$. In Section 6, the moment method will be considered.

Now we describe the FMM. As said earlier, the cost of a matrix-vector multiplication is $O\left(n^{2}\right)$, where $n$ is the size of the dense matrix. But for some special forms of matrices, this cost can be decreased a lot. Let us consider an $n \times n$ matrix $\mathbf{A}$ whose elements take the form $\mathbf{A}_{i j}=\alpha_{i} \beta_{j}$, for some complex numbers $\alpha_{1}, \cdots, \alpha_{n}$, and $\beta_{1}, \cdots, \beta_{n}$. Then the multiplication of the matrix $\mathbf{A}$ by any vector $\left[\begin{array}{c}x_{1} \\ \vdots \\ x_{n}\end{array}\right]$ can be done in a fast manner since

$$
\mathbf{A} \cdot\left[\begin{array}{c}
x_{1} \\
\vdots \\
x_{n}
\end{array}\right]=\left[\begin{array}{c}
\alpha_{1} y \\
\vdots \\
\alpha_{n} y
\end{array}\right], \quad \text { where } y=\sum_{i=1}^{n} \beta_{i} x_{i}
$$

Hence it requires only $2 n$ operations by using the following method: the calculation of $y$ is done only once and is used $n$ times. So the first idea to perform a fast matrixvector multiplication can be stated as follows: some preliminary calculations are done only once, then stored and used many times. The example of the matrix $\mathbf{A}$ also motivates the second idea of the FMM: The terms $\mathbf{Z}_{i j}$ of the matrix $\mathbf{Z}$ must be written as $\alpha_{i} \beta_{j}$. Whatever the discretization may be, this means that the kernel $G\left(\mathrm{x}, \mathrm{x}^{\prime}\right)$ must be written as (or approximated by) a function of $\mathrm{x}$ times a function of $\mathrm{x}^{\prime}$, for all $\mathrm{x}, \mathrm{x}^{\prime} \in \Gamma$. Unfortunately, such a global representation (or approximation) cannot be found. But we will show that $G\left(\mathbf{x}, \mathbf{x}^{\prime}\right)$ can be locally approximated by a function of $\mathbf{x}$ times a function of $\mathbf{x}^{\prime}$. More precisely, if $A_{i}$ and $A_{j}$ are two different subsets of the boundary $\Gamma$, an approximate form of $G\left(\mathbf{x}, \mathbf{x}^{\prime}\right)$ will be given for all $\mathbf{x} \in A_{i}$ and $\mathbf{x}^{\prime} \in A_{j}$. But the expression of the approximation will be different for different sets $A_{i}$ and $A_{j}$.

Let us now introduce some notation in order to give the approximate expression of the kernel $G . \Gamma$ is split into a cluster of say, $p$ subsets $A_{1} \ldots A_{p}: \Gamma=\bigcup_{i=1}^{p} A_{i}$, with $A_{i} \cap A_{j}=\emptyset$ if $i \neq j$. These subsets are referred to as "aggregates". For the aggregate $A_{i}$, a reference point $\mathbf{z}_{i}$ is introduced. It is located roughly at the center of the curve $A_{i}$. The point $\mathbf{z}_{i}$ may or not be on the boundary $\Gamma$ (see Figure 1). 


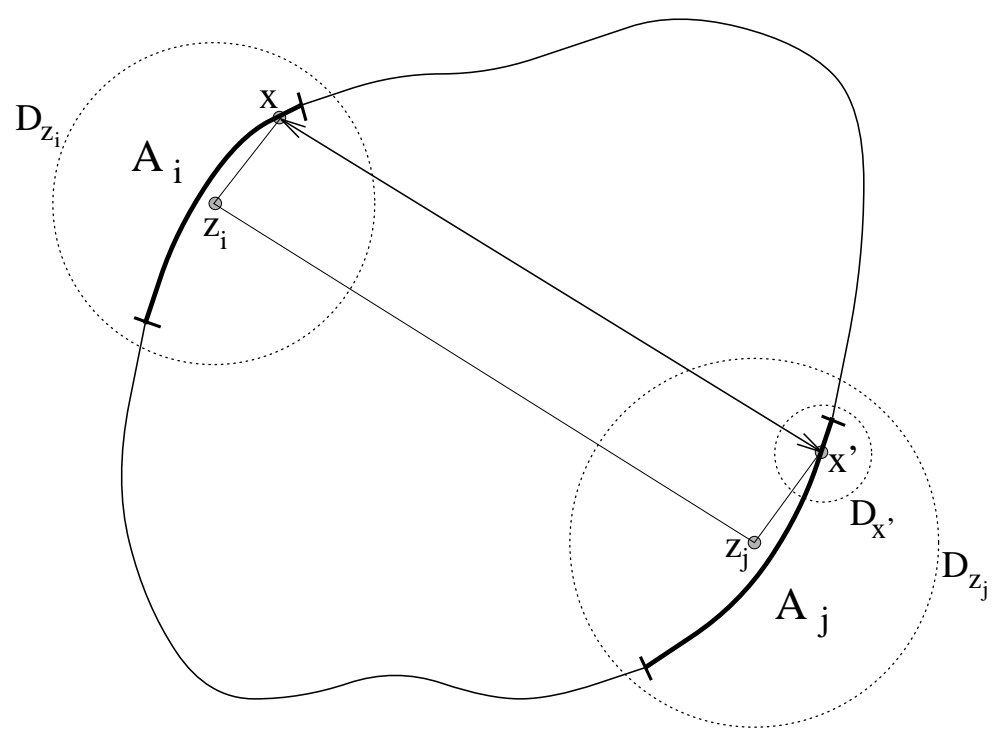

Figure 1. Location of the center $\mathbf{z}_{i}$ of $A_{i}$.

Let $\mathbf{U}(\theta)$ be the vector of $\mathbb{R}^{2}$ equal to $\left(\begin{array}{c}\cos \theta \\ \sin \theta\end{array}\right)$. Then for $\mathbf{x} \in A_{i}$ and $\mathbf{x}^{\prime} \in A_{j}$, the kernel is approximated by the formula

$$
\begin{aligned}
G\left(\mathbf{x}, \mathbf{x}^{\prime}\right) \approx G_{N}^{\mathcal{C}}\left(\mathbf{x}, \mathbf{x}^{\prime}\right):=\frac{1}{4 \pi^{2}} \int_{0}^{2 \pi} \exp \left(\imath k\left(\mathbf{x}-\mathbf{z}_{i}\right) \cdot \mathbf{U}(\theta)\right) \exp \left(-\imath k\left(\mathbf{x}^{\prime}-\mathbf{z}_{j}\right) \cdot \mathbf{U}(\theta)\right) \\
\quad \cdot\left[\sum_{m=-N}^{N} \exp \left(\imath m\left(\theta-\arg \left(\mathbf{z}_{i}-\mathbf{z}_{j}\right)\right)\right) K_{|m|}\left(-\imath k \mathbf{z}_{i}-\mathbf{z}_{j}\right)\right] d \theta
\end{aligned}
$$

for a suitable choice of $N \in \mathbb{N}^{*}$ and $i, j$. The approximate kernel $G_{N}^{\mathcal{C}}$ is referred to as the continuous approximated kernel. Obviously, $G_{N}^{\mathcal{C}}$ is well defined as soon as $i \neq j$. Some quick numerical simulations can persuade the reader that the approximation in (4) is very accurate, for a suitable choice of $N \in \mathbb{N}^{*}$ and $i, j$. The purpose of Theorem 2 in Section 4 is to give the values of $i$ and $j$ for which formula (4) holds, and to give in this case an estimate of the error. Theorem 2 also explains the meaning of the sign $\approx$ in $(4)$.

The term in brackets in the right hand side of (4) does not converge absolutely as $N \rightarrow \infty$. Thus the limit of the right hand side of (4) when $N \rightarrow \infty$ must be taken in Cesaro's sense. This means that the right hand side of (4) converges but the limit is not equal to the value

$$
\begin{aligned}
\frac{1}{4 \pi^{2}} \int_{0}^{2 \pi} \exp \left(\imath k\left(\mathbf{x}-\mathbf{z}_{i}\right) \cdot \mathbf{U}(\theta)\right) \exp \left(-\imath k\left(\mathbf{x}^{\prime}-\mathbf{z}_{j}\right) \cdot \mathbf{U}(\theta)\right) \\
\cdot\left[\sum_{m \in \mathbb{Z}} \exp \left(\imath m\left(\theta-\arg \left(\mathbf{z}_{i}-\mathbf{z}_{j}\right)\right)\right) K_{|m|}\left(-\imath k \mid \mathbf{z}_{i}-\mathbf{z}_{j}\right)\right] d \theta
\end{aligned}
$$

since this last term is not defined.

Let us notice that the approximate kernel in (4) takes roughly the following form: a function of $\mathbf{x}$ times a function of $\mathbf{x}^{\prime}$. In fact, we also have an integration 
over $[0,2 \pi]$. We take care of this integration right now. Obviously, for numerical considerations, the integral must be discretized. For this, we use the trapezoidal rule: for any function $F$, we write

$$
\frac{1}{2 \pi} \int_{0}^{2 \pi} F(\theta) d \theta \approx \frac{1}{N_{T}} \sum_{n_{T}=1}^{N_{T}} F\left(\theta_{n_{T}}\right),
$$

where $\theta_{n_{T}}=\frac{2 \pi}{N_{T}} n_{T}$, and

$$
N_{T}=2 N+1
$$

This reason of this choice will become clear in Section 5 . Hence we also have

$$
\begin{aligned}
G\left(\mathbf{x}, \mathbf{x}^{\prime}\right) \approx G_{N}^{\mathcal{D}}\left(\mathbf{x}, \mathbf{x}^{\prime}\right):=\frac{1}{2 \pi N_{T}} \sum_{n_{T}=1}^{N_{T}} \exp \left(\imath k\left(\mathbf{x}-\mathbf{z}_{i}\right) \cdot \mathbf{U}\left(\theta_{n_{T}}\right)\right) \\
\cdot \exp \left(-\imath k\left(\mathbf{x}^{\prime}-\mathbf{z}_{j}\right) \cdot \mathbf{U}\left(\theta_{n_{T}}\right)\right) \\
\cdot\left[\sum_{m=-N}^{N} \exp \left(\imath m\left(\theta_{n_{T}}-\arg \left(\mathbf{z}_{i}-\mathbf{z}_{j}\right)\right)\right) K_{|m|}\left(-\imath k \mid \mathbf{z}_{i}-\mathbf{z}_{j}\right)\right] .
\end{aligned}
$$

$G_{N}^{\mathcal{D}}$ is referred to as the discretized approximated kernel. (6) will be proved to hold under the same sense as (4). Actually the proof of convergence of (6) is not done just by combining (4) and a theorem of convergence of the summation formula. The fact that $N_{T}$ is fixed at $2 N+1$ makes the convergence analysis a little more subtle.

\section{Integral APPRoXimation OF the HANKEL FUNCTION}

The purpose of this section is to give a quick justification of (4). But the sense of the sign " $\approx$ " will remain indefinite at the end of this section. The calculation of the limit value of the right hand side of (4) is postponed to the next section (see Lemma 1, Theorem 2 and Corollary 3).

Let us consider two different subsets $A_{i}$ and $A_{j}$ of $\Gamma$. Basically, we are interested in deriving an approximated form of $G\left(\mathbf{x}, \mathbf{x}^{\prime}\right)$ for all $\mathbf{x} \in A_{i}$ and $\mathbf{x}^{\prime} \in A_{j}$. In this section let us fix $\mathbf{x}^{\prime} \in A_{j}$ and let $D_{\mathbf{z}_{i}}, D_{\mathbf{x}^{\prime}}, D_{\mathbf{z}_{j}}$ be three balls with centers respectively $\mathbf{z}_{i}, \mathbf{x}^{\prime}$ and $\mathbf{z}_{j}$ (see Figure 1 ) chosen so that $D_{\mathbf{x}^{\prime}} \subset D_{\mathbf{z}_{j}}$ and $D_{\mathbf{z}_{i}} \cap D_{\mathbf{z}_{j}}=\emptyset$.

With the help of the two Propositions 9 and 10 (see Section 10 in the Appendix), we are able to give another form of the kernel. At this point, the exact hypothesis on $i$ and $j$ remains indefinite. As noticed before, we must at least assume that $i \neq j$. Let us compute for $\mathbf{x} \in D_{\mathbf{z}_{i}}$ the function

$$
G\left(\mathbf{x}, \mathbf{x}^{\prime}\right)=\frac{\imath}{4} H_{0}^{(1)}\left(k\left|\mathbf{x}-\mathbf{x}^{\prime}\right|\right)=\frac{\imath}{4} \sum_{m \in \mathbb{Z}} \beta_{m} H_{m}^{(1)}\left(k\left|\mathbf{x}-\mathbf{x}^{\prime}\right|\right) \exp \left(\imath m \arg \left(\mathbf{x}-\mathbf{x}^{\prime}\right)\right)
$$

with $\beta_{0}=1$ and $\beta_{m}=0$ if $m \neq 0$.

In order to use part (i) of Proposition 9, we have to verify the two assumptions given in Proposition 9. First, $\tilde{\beta}_{m}$ (defined in Proposition 9) equals

$$
\tilde{\beta}_{m}=\exp \left(-\imath m \arg \left(\mathbf{x}^{\prime}-\mathbf{z}_{j}\right)\right) J_{m}\left(k\left|\mathbf{x}^{\prime}-\mathbf{z}_{j}\right|\right) .
$$

In addition, thanks to (36) and (37), one may write for $|m|$ large enough

$$
\left|\tilde{\beta}_{m} H_{m}^{(1)}\left(k\left|\mathbf{x}-\mathbf{z}_{j}\right|\right)\right| \leq \frac{M_{J} M_{H}}{\pi|m|}\left(\frac{\left|\mathbf{x}^{\prime}-\mathbf{z}_{j}\right|}{\left|\mathbf{x}-\mathbf{z}_{j}\right|}\right)^{|m|} .
$$


Since $\mathbf{x}^{\prime} \in D_{\mathbf{z}_{j}}$ and $\mathbf{x} \notin D_{z_{j}}$, we have $\frac{\left|\mathbf{x}^{\prime}-\mathbf{z}_{j}\right|}{\left|\mathbf{x}-\mathbf{z}_{j}\right|}<1$. Consequently,

$$
\sum_{m \in \mathbb{Z}}\left|\tilde{\beta}_{m} H_{m}^{(1)}\left(k\left|\mathbf{x}-\mathbf{z}_{j}\right|\right)\right|
$$

converges. Therefore by part (i) of Proposition 9, one can write

$$
G\left(\mathbf{x}, \mathbf{x}^{\prime}\right)=\frac{\imath}{4} \sum_{m \in \mathbb{Z}} \tilde{\beta}_{m} H_{m}^{(1)}\left(k\left|\mathbf{x}-\mathbf{z}_{j}\right|\right) \exp \left(\imath m \arg \left(\mathbf{x}-\mathbf{z}_{j}\right)\right) .
$$

Let us now use part (ii) of Proposition 9. The convergence of

$$
\sum_{l}\left|\tilde{\beta}_{m-l} H_{l}^{(1)}\left(k\left|\mathbf{z}_{i}-\mathbf{z}_{j}\right|\right)\right|
$$

will be shown in Lemma 1. Hence one can define

$$
\gamma_{m}:=\sum_{l \in \mathbb{Z}} \exp \left(-\imath l\left(\arg \left(\mathbf{z}_{i}-\mathbf{z}_{j}\right)-\pi\right)\right) \tilde{\beta}_{m-l} H_{l}^{(1)}\left(k\left|\mathbf{z}_{i}-\mathbf{z}_{j}\right|\right) .
$$

Moreover, by Corollary 3 , the expansion $\sum_{m \in \mathbb{Z}}\left|\gamma_{m} J_{m}\left(k\left|\mathbf{x}-\mathbf{z}_{i}\right|\right)\right|$ converges. Then using Proposition 9, part (ii), we get

$$
G\left(\mathbf{x}, \mathbf{x}^{\prime}\right)=\frac{\imath}{4} \sum_{m \in \mathbb{Z}} \gamma_{m} J_{m}\left(k\left|\mathbf{x}-\mathbf{z}_{i}\right|\right) \exp \left(\imath m \arg \left(\mathbf{x}-\mathbf{z}_{i}\right)\right) .
$$

Now we want to use Proposition 10. To satisfy the condition of Proposition 10, the expansion in the definition of $\gamma_{m}$ must be truncated. Consequently let us define $\left(\right.$ for $N \in \mathbb{N}^{*}$ )

$$
\gamma_{m}^{(N)}:=\sum_{l=-N}^{N} \exp \left(-\imath l\left(\arg \left(\mathbf{z}_{i}-\mathbf{z}_{j}\right)-\pi\right)\right) \tilde{\beta}_{m-l} H_{l}^{(1)}\left(k\left|\mathbf{z}_{i}-\mathbf{z}_{j}\right|\right) .
$$

One can indeed show that $\sum_{m} \gamma_{m} \exp (\imath m(\theta-\pi / 2))$ does not converge absolutely whereas the expansion $\sum_{m} \gamma_{m}^{(N)} \exp (\imath m(\theta-\pi / 2))$ converges absolutely. The proof of this latter result is also postponed to Lemma 1 . Thus

$$
G\left(\mathbf{x}, \mathbf{x}^{\prime}\right) \approx G_{N}^{\mathcal{C}}\left(\mathbf{x}, \mathbf{x}^{\prime}\right):=\frac{\imath}{4} \sum_{m \in \mathbb{Z}} \gamma_{m}^{(N)} J_{m}\left(k\left|\mathbf{x}-\mathbf{z}_{i}\right|\right) \exp \left(\imath m \arg \left(\mathbf{x}-\mathbf{z}_{i}\right)\right) .
$$

As a consequence of Proposition 10, we have

$$
\begin{aligned}
G_{N}^{\mathcal{C}}\left(\mathbf{x}, \mathbf{x}^{\prime}\right)=\frac{\imath}{8 \pi} \int_{0}^{2 \pi} \exp \left(\imath k\left|\mathbf{x}-\mathbf{z}_{i}\right| \cos \left(\theta-\arg \left(\mathbf{x}-\mathbf{z}_{i}\right)\right)\right) \\
\cdot\left[\sum_{m \in \mathbb{Z}} \gamma_{m}^{(N)} \exp (\imath m \theta) \exp (-\imath m \pi / 2)\right] d \theta .
\end{aligned}
$$

Let us focus on the term in brackets,

$$
\begin{aligned}
& \sum_{m \in \mathbb{Z}} \gamma_{m}^{(N)} \exp (\imath m \theta) \exp (-\imath m \pi / 2) \\
& \quad=\sum_{m \in \mathbb{Z}} \sum_{l=-N}^{N} \exp \left(-\imath l\left(\arg \left(\mathbf{z}_{i}-\mathbf{z}_{j}\right)-\pi\right)\right) \tilde{\beta}_{m-l} H_{l}^{(1)}\left(k\left|\mathbf{z}_{i}-\mathbf{z}_{j}\right|\right) \exp (\imath m(\theta-\pi / 2))
\end{aligned}
$$


By setting $t=m-l$ and $s=l$, we have

$$
\begin{aligned}
\sum_{m \in \mathbb{Z}} \gamma_{m}^{(N)} \exp (\imath m \theta) \exp (-\imath m \pi / 2)=\left(\sum_{t \in \mathbb{Z}} \tilde{\beta}_{t} \exp (\imath t(\theta-\pi / 2))\right) \\
\cdot\left(\sum_{s=-N}^{N} H_{s}^{(1)}\left(k\left|\mathbf{z}_{i}-\mathbf{z}_{j}\right|\right) \exp \left(-\imath s\left(\pi / 2-\theta+\arg \left(\mathbf{z}_{i}-\mathbf{z}_{j}\right)-\pi\right)\right)\right) .
\end{aligned}
$$

The first expansion in the right hand side converges and is equal to

$$
\sum_{t \in \mathbb{Z}} \tilde{\beta}_{t} \exp (\imath t(\theta-\pi / 2))=\sum_{t \in \mathbb{Z}} \exp \left(\imath t\left(-\pi / 2+\theta-\arg \left(\mathbf{x}^{\prime}-\mathbf{z}_{j}\right)\right)\right) J_{t}\left(k\left|\mathbf{x}^{\prime}-\mathbf{z}_{j}\right|\right) .
$$

With (45), it follows that

$$
\begin{aligned}
\sum_{t \in \mathbb{Z}} \tilde{\beta}_{t} \exp (\imath t(\theta-\pi / 2)) & =\exp \left(\imath k\left|\mathbf{x}^{\prime}-\mathbf{z}_{j}\right| \sin \left(-\pi / 2+\theta-\arg \left(\mathbf{x}^{\prime}-\mathbf{z}_{j}\right)\right)\right) \\
& =\exp \left(-\imath k\left|\mathbf{x}^{\prime}-\mathbf{z}_{j}\right| \cos \left(\theta-\arg \left(\mathbf{x}^{\prime}-\mathbf{z}_{j}\right)\right)\right) .
\end{aligned}
$$

Finally

$$
\begin{aligned}
G\left(\mathbf{x}, \mathbf{x}^{\prime}\right) \approx G_{N}^{\mathcal{C}}\left(\mathbf{x}, \mathbf{x}^{\prime}\right) \\
=\frac{\imath}{4} \frac{1}{2 \pi} \int_{0}^{2 \pi} \exp \left(\imath k\left|\mathbf{x}-\mathbf{z}_{i}\right| \cos \left(\theta-\arg \left(\mathbf{x}-\mathbf{z}_{i}\right)\right)\right) \\
\cdot \exp \left(-\imath k\left|\mathbf{x}^{\prime}-\mathbf{z}_{j}\right| \cos \left(\theta-\arg \left(\mathbf{x}^{\prime}-\mathbf{z}_{j}\right)\right)\right) \\
\cdot\left[\sum_{s=-N}^{N} H_{s}^{(1)}\left(k\left|\mathbf{z}_{i}-\mathbf{z}_{j}\right|\right) \exp \left(-\imath s\left(-\pi / 2-\theta+\arg \left(\mathbf{z}_{i}-\mathbf{z}_{j}\right)\right)\right)\right] d \theta .
\end{aligned}
$$

A straightforward calculation shows that

$$
\begin{aligned}
& G\left(\mathbf{x}, \mathbf{x}^{\prime}\right) \approx G_{N}^{\mathcal{C}}\left(\mathbf{x}, \mathbf{x}^{\prime}\right) \\
& \quad=\frac{1}{2 \pi} \int_{0}^{2 \pi} \frac{1}{2 \pi} \exp \left(\imath k\left(\mathbf{x}-\mathbf{z}_{i}\right) \cdot \mathbf{U}(\theta)\right) \exp \left(-\imath k\left(\mathbf{x}^{\prime}-\mathbf{z}_{j}\right) \cdot \mathbf{U}(\theta)\right) \tau_{i j}^{(N)}(\theta) d \theta
\end{aligned}
$$

where

$$
\tau_{i j}^{(N)}(\theta):=\sum_{m=-N}^{N} \exp \left(-\imath m\left(-\pi / 2-\theta+\arg \left(\mathbf{z}_{i}-\mathbf{z}_{j}\right)\right)\right)(-\imath)^{m} K_{m}\left(-\imath k\left|\mathbf{z}_{i}-\mathbf{z}_{j}\right|\right) .
$$

By using both the definition of the modified Hankel function and the formula (35), we have

$$
K_{-|m|}(-\imath z)=\frac{\imath \pi}{2} \imath^{-|m|} H_{-|m|}^{(1)}(z)=\frac{\imath \pi}{2}(-\imath)^{|m|}(-1)^{|m|} H_{|m|}(z)=K_{|m|}(-\imath z) .
$$

Hence

$$
\tau_{i j}^{(N)}(\theta)=\sum_{m=-N}^{N} \exp \left(\imath m\left(\theta-\arg \left(\mathbf{z}_{i}-\mathbf{z}_{j}\right)\right)\right) K_{|m|}\left(-\imath k \mid \mathbf{z}_{i}-\mathbf{z}_{j \mid}\right)
$$

By putting together (9) and (10), we get (4). 


\section{Convergence AnAlysis of the CONTINuOUs APPROXimated Kernel}

This section is devoted to proving the convergence of the approximation (4) as $N \rightarrow \infty$, i.e. the error

$$
G\left(\mathbf{x}, \mathbf{x}^{\prime}\right)-G_{N}^{\mathcal{C}}\left(\mathbf{x}, \mathbf{x}^{\prime}\right)=\frac{\imath}{4} \sum_{m \in \mathbb{Z}}\left(\gamma_{m}-\gamma_{m}^{(N)}\right) J_{m}\left(k \mid \mathbf{x}-\mathbf{z}_{i}\right) \exp \left(\imath m \arg \left(\mathbf{x}-\mathbf{z}_{i}\right)\right)
$$

when $N \rightarrow \infty$. More generally, all the calculations of the previous section are justified here.

The next lemma proves the convergence of the expansion $\gamma_{m}$ and gives an upper bound of the term $\left|\gamma_{m}-\gamma_{m}^{(N)}\right|$. In this section we assume that $\mathbf{x} \in A_{i}$ and $\mathbf{x}^{\prime} \in A_{j}$, with $i, j$ so chosen that $A_{i} \subset D_{\mathbf{z}_{i}}$ and $A_{j} \subset D_{\mathbf{z}_{j}}$ (where the balls $D_{\mathbf{z}_{i}}, D_{\mathbf{z}_{j}}$ satisfy the requirement of the previous section). Moreover, in the rest of this paper, we will use the estimates (36) and (37) with the choice $R=|k| \max _{i, j}\left|\mathbf{z}_{i}-\mathbf{z}_{j}\right|$ and $R^{\prime}=|k| \min _{i, j}\left|\mathbf{z}_{i}-\mathbf{z}_{j}\right|$.

Lemma 1. Let $\Lambda$ be the smallest integer greater than the following four numbers:

$$
\begin{aligned}
C|k|^{2} \max _{i, j}\left|\mathbf{z}_{i}-\mathbf{z}_{j}\right|^{2}, & \frac{\mathrm{e}}{2}|k| \max _{i, j}\left|\mathbf{z}_{i}-\mathbf{z}_{j}\right|, \\
C|k|^{2} \max _{i} \max _{\mathbf{x} \in A_{i}}\left|\mathbf{x}-\mathbf{z}_{i}\right|^{2}, & \frac{\mathrm{e}}{2}|k| \max _{i} \max _{\mathbf{x} \in A_{i}}\left|\mathbf{x}-\mathbf{z}_{i}\right|,
\end{aligned}
$$

where $C$ is given in Proposition 8. We assume that $\gamma_{m}^{(N)}$ is given by (8) and $\tilde{\beta}_{m}$ by (7). Let $A_{i}$ and $A_{j}$ be two aggregates of $\Gamma$ such that $i \neq j$. We consider $\mathbf{x} \in A_{i}$ and $\mathbf{x}^{\prime} \in A_{j}$ such that $\frac{\left|\mathbf{x}-\mathbf{z}_{i}\right|}{\mathbf{z}_{i}-\mathbf{z}_{j} \mid}<1$ and $\frac{\left|\mathbf{x}^{\prime}-\mathbf{z}_{j}\right|}{\mathbf{z}_{i}-\mathbf{z}_{j} \mid}<\frac{1}{\sqrt{\mathrm{e}}}$. Then

- $\lim _{N \rightarrow \infty} \gamma_{m}^{(N)}$ exists (we have convergence in norm) and the limit is equal to $\gamma_{m}$.

- The expansion $\sum_{m \in \mathbb{Z}} \gamma_{m}^{(N)} \exp (\imath m(\theta-\pi / 2))$ converges absolutely for all $\theta \in$ $\mathbb{R}$.

- Assume now that $N>\Lambda$. If $|m| \leq N-\Lambda$, we have

$$
\left|\gamma_{m}-\gamma_{m}^{(N)}\right| \leq C^{(-)}\left(\frac{4 N}{|k|\left|\mathbf{x}^{\prime}-\mathbf{z}_{j}\right|}\right)^{|m|} \frac{\left(\frac{\left|\mathbf{x}^{\prime}-\mathbf{z}_{j}\right|}{\left|\mathbf{z}_{i}-\mathbf{z}_{j}\right|}\right)^{N}}{\left(1-\frac{\left|\mathbf{x}^{\prime}-\mathbf{z}_{j}\right|}{\left|\mathbf{z}_{i}-\mathbf{z}_{j}\right|}\right)^{|m|}}
$$

and, if $|m|>N-\Lambda$, then

$$
\begin{aligned}
\left|\gamma_{m}-\gamma_{m}^{(N)}\right| \leq & C_{1}^{(+)}\left(\frac{2(m \mid+\Lambda)}{\mathrm{e}|k|\left|\mathbf{z}_{i}-\mathbf{z}_{j}\right|}\right)^{|m|+\Lambda} \\
& +C_{2}^{(+)} \sqrt{m}\left(\frac{4|m|}{\mathrm{e}|k|\left|\mathbf{x}^{\prime}-\mathbf{z}_{j}\right|}\right)^{|m|} \frac{\left(\mathrm{e} \frac{\left|\mathbf{x}^{\prime}-\mathbf{z}_{j}\right|}{\mathbf{z}_{i}-\mathbf{z}_{j} \mid}\right)^{|m|+\Lambda}}{\left(1-\frac{\left|\mathbf{x}^{\prime}-\mathbf{z}_{j}\right|}{\left|\mathbf{z}_{i}-\mathbf{z}_{j}\right|}\right)^{|m|}}
\end{aligned}
$$

where $C^{(-)}, C_{1}^{(+)}$and $C_{2}^{(+)}$are some constants which only depend on $\Lambda$. 
Proof. With (7) and (36), $\tilde{\beta}_{m}$ satisfies for $|m|>C|k|^{2}\left|\mathbf{x}^{\prime}-\mathbf{z}_{j}\right|^{2}$, the inequality

$$
\left|\tilde{\beta}_{m}\right| \leq \frac{M_{J}}{\sqrt{2 \pi|m|}}\left(\frac{\mathrm{e}|k|\left|\mathbf{x}^{\prime}-\mathbf{z}_{j}\right|}{2|m|}\right)^{|m|} .
$$

Thanks to the definition of $\Lambda$, this relation holds in particular when $|m|>\Lambda$.

Let us set $\Delta_{l}^{(m)}:=\exp \left(-\imath l\left(\arg \left(\mathbf{z}_{i}-\mathbf{z}_{j}\right)-\pi\right)\right) \tilde{\beta}_{m-l} H_{l}^{(1)}\left(k\left|\mathbf{z}_{i}-\mathbf{z}_{j}\right|\right)$. This is the general term in the expansion of $\gamma_{m}$ or $\gamma_{m}^{(N)}$.

- In order to study the convergence of $\gamma_{m}^{(N)}$ as $N \rightarrow \infty$, let us fix $m \in \mathbb{N}$ and assume that $N>m+\Lambda$. We have $\gamma_{m}^{(N)}=\sum_{l=-N}^{N} \Delta_{l}^{(m)}$. Consider first the case when $l>m+\Lambda$. By (37) and (13), we have

$$
\left|\Delta_{l}^{(m)}\right| \leq M_{H} \sqrt{\frac{2}{\pi l}}\left(\frac{2 l}{\mathrm{e}|k| \mathbf{z}_{i}-\mathbf{z}_{j} \mid}\right)^{l} \frac{M_{J}}{\sqrt{2 \pi(l-m)}}\left(\frac{\mathrm{e}|k|\left|\mathbf{x}^{\prime}-\mathbf{z}_{j}\right|}{2(l-m)}\right)^{l-m},
$$

which leads to

$$
\left|\Delta_{l}^{(m)}\right| \leq \frac{M_{J} M_{H}}{\pi \sqrt{l(l-m)}}\left(\frac{2}{\mathrm{e}|k|\left|\mathbf{x}^{\prime}-\mathbf{z}_{j}\right|}\right)^{m} \frac{l^{l}}{(l-m)^{l-m}}\left(\frac{\left|\mathbf{x}^{\prime}-\mathbf{z}_{j}\right|}{\left|\mathbf{z}_{i}-\mathbf{z}_{j}\right|}\right)^{l} .
$$

Using the relation $\frac{1}{\sqrt{l(l-m)}} \leq 1$ and Proposition 11 part $(i)$, we get

$$
\left|\Delta_{l}^{(m)}\right| \leq \frac{M_{J} M_{H}}{\pi}\left(\frac{2}{|k|\left|\mathbf{x}^{\prime}-\mathbf{z}_{j}\right|}\right)^{m} l^{m}\left(\frac{\left|\mathbf{x}^{\prime}-\mathbf{z}_{j}\right|}{\left|\mathbf{z}_{i}-\mathbf{z}_{j}\right|}\right)^{l} \text { if } l>m+\Lambda .
$$

Since $\frac{\left|\mathbf{x}^{\prime}-\mathbf{z}_{j}\right|}{\mathbf{z}_{i}-\mathbf{z}_{j} \mid}<1$, the expansion $\sum_{l} l^{m}\left(\frac{\left|\mathbf{x}^{\prime}-\mathbf{z}_{j}\right|}{\left|\mathbf{z}_{i}-\mathbf{z}_{j}\right|}\right)^{l}$ is convergent, which proves that the expansion $\sum_{l \geq m+\Lambda} \Delta_{l}^{(m)}$ converges absolutely.

Now, for $l<-(m+\Lambda)$, we arrive at

$$
\begin{aligned}
\left|\Delta_{l}^{(m)}\right| & \leq \frac{M_{J} M_{H}}{\pi \sqrt{|l|(l \mid+m)}}\left(\frac{\mathrm{e}|k|\left|\mathbf{x}^{\prime}-\mathbf{z}_{j}\right|}{2(l l \mid+m)}\right)^{|l|+m}\left(\frac{2|l|}{\mathrm{e}|k| \mathbf{z}_{i}-\mathbf{z}_{j} \mid}\right)^{|l|} \\
& \leq \frac{M_{J} M_{H}}{\pi \sqrt{|l|(l l+m)}}\left(\frac{\mathrm{e}|k|\left|\mathbf{x}^{\prime}-\mathbf{z}_{j}\right|}{2}\right)^{m} \frac{|l|^{|l|}}{(l \mid+m)^{|l|+m}}\left(\frac{\left|\mathbf{x}^{\prime}-\mathbf{z}_{j}\right|}{\left|\mathbf{z}_{i}-\mathbf{z}_{j}\right|}\right)^{|l|} .
\end{aligned}
$$

By Proposition 11 part (ii), we have $\frac{|l|^{|l|}}{(l \mid+m)^{|l|+m}} \leq \mathrm{e}^{-m}|l|^{-m}$. Therefore

$$
\left|\Delta_{l}^{(m)}\right| \leq \frac{M_{J} M_{H}}{\pi}\left(\frac{|k|\left|\mathbf{x}^{\prime}-\mathbf{z}_{j}\right|}{2}\right)^{m} \frac{1}{|l|^{m}}\left(\frac{\left|\mathbf{x}^{\prime}-\mathbf{z}_{j}\right|}{\left|\mathbf{z}_{i}-\mathbf{z}_{j}\right|}\right)^{|l|} \text { if } l<-(m+\Lambda) .
$$

Thus by using the same arguments as above, we can infer that $\sum_{l \leq-(m+\Lambda)} \Delta_{l}^{(m)}$ converges absolutely. Hence, $\lim _{N \rightarrow \infty} \gamma_{m}^{(N)}$ exists and is equal to $\sum_{l \in \mathbb{Z}} \Delta_{l}^{(m)}=$ $\gamma_{m}$.

- We now want to study the convergence of $\sum_{m \in \mathbb{Z}} \gamma_{m}^{(N)} \exp (\imath m(\theta-\pi / 2))$ for $N$ fixed. To this end, let us consider $m \geq N+\Lambda$. We set

$$
\mathcal{H}_{N}:=\max _{-N \leq l \leq N}\left|H_{l}^{(1)}\left(k\left|z_{i}-z_{j}\right|\right)\right| \text {. }
$$

Hence

$$
\left|\gamma_{m}^{(N)}\right| \leq \sum_{l=-N}^{N}\left|\tilde{\beta}_{m-l}\right|\left|H_{l}^{(1)}\left(k\left|\mathbf{z}_{i}-\mathbf{z}_{j}\right|\right)\right| \leq(2 N+1) \mathcal{H}_{N} \max _{l=-N, \ldots, N}\left|\tilde{\beta}_{m-l}\right| .
$$




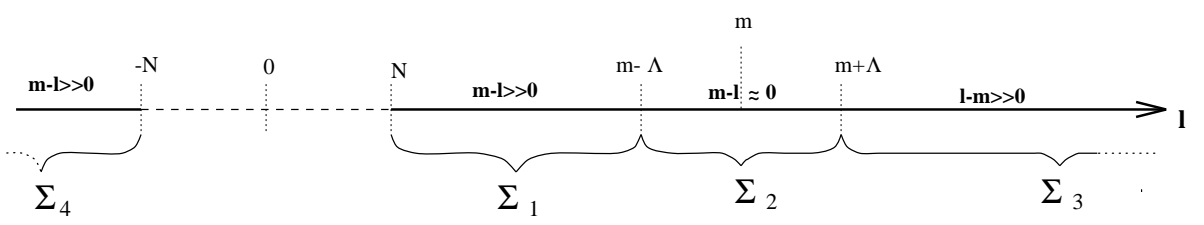

FiguRE 2. Location of the four sets, for $m \in \mathbb{Z}$ fixed.

Setting $l^{\prime}=m-l$, relation (13) yields

$$
\max _{l=-N, \ldots, N}\left|\tilde{\beta}_{m-l}\right| \leq \frac{M_{J}}{\sqrt{2 \pi}} \max _{m-N \leq l^{\prime} \leq m+N}\left\{\left(\frac{\mathrm{e}|k|\left|\mathbf{x}^{\prime}-\mathbf{z}_{j}\right|}{2 l^{\prime}}\right)^{l^{\prime}}\right\} .
$$

We apply Proposition 12 part (i) to $x=l^{\prime}, a=\frac{\mathrm{e}}{2}|k|\left|\mathbf{x}^{\prime}-\mathbf{z}_{j}\right|$ and $y=m-N$. Since $x \geq y=m-N \geq \Lambda \geq \frac{\mathrm{e}}{2}|k|\left|\mathbf{x}^{\prime}-\mathbf{z}_{j}\right|=a$, we have

$$
\max _{l=-N, \ldots, N}\left|\tilde{\beta}_{m-l}\right| \leq \frac{M_{J}}{\sqrt{2 \pi}}\left(\frac{\mathrm{e}|k|\left|\mathbf{x}^{\prime}-\mathbf{z}_{j}\right|}{2(m-N)}\right)^{m-N} .
$$

Hence

$$
\left|\gamma_{m}^{(N)}\right| \leq \frac{M_{J}(2 N+1)}{\sqrt{2 \pi}} \mathcal{H}_{N}\left(\frac{\mathrm{e}|k| \mathbf{x}^{\prime}-\mathbf{z}_{j} \mid}{2(m-N)}\right)^{m-N}
$$

Since $N$ is fixed, we conclude that $\sum_{m=0}^{\infty}\left|\gamma_{m}^{(N)}\right|<\infty$. Similarly, the convergence for the negative values of $m$ can be shown. Therefore,

$$
\sum_{m \in \mathbb{Z}} \gamma_{m}^{(N)} \exp (\imath m(\theta-\pi / 2))
$$

converges uniformly for any $\theta$.

- It remains to show the inequalities (11) and (12). Without loss of generality, we assume that $m$ is a nonnegative integer. The case $m<0$ can be deduced from the case $m \geq 0$, thanks to formula (35). We now assume that $N>\Lambda$.

First, one notes that the truncation error is

$$
\gamma_{m}-\gamma_{m}^{(N)}=\sum_{|l|>N} \exp \left(-\imath l\left(\arg \left(\mathbf{z}_{i}-\mathbf{z}_{j}\right)-\pi\right)\right) \tilde{\beta}_{m-l} H_{l}^{(1)}\left(k\left|\mathbf{z}_{i}-\mathbf{z}_{j}\right|\right)=\sum_{|l|>N} \Delta_{l}^{(m)} .
$$

In the last sum, the term $H_{l}^{(1)}\left(k\left|\mathbf{z}_{i}-\mathbf{z}_{j}\right|\right)$ is considered for $|l|>N>\Lambda$, so that it can always be bounded with the help of inequality (37). Formula (13) enables one to bound $\tilde{\beta}_{m-l}$ when $|m-l|>\Lambda$. Finally, the two cases $l \geq 0$ and $l<0$ must be taken into account. Due to these remarks, the set $\Sigma:=\{l \in \mathbb{Z},|l|>N\}$ is split into four sets, as depicted in Figure 2: $\Sigma_{1}:=$ $\{l \in \mathbb{Z}, N<l \leq m-\Lambda\} \cap \Sigma, \Sigma_{2}:=\{l \in \mathbb{Z}, m-\Lambda<l \leq m+\Lambda\} \cap \Sigma, \Sigma_{3}:=$ $\{l \in \mathbb{Z}, l>m+\Lambda\} \cap \Sigma$, and $\Sigma_{4}:=\{l \in \mathbb{Z}, l<-N\}$.

When $0 \leq m \leq N-\Lambda$, the sets $\Sigma_{1}$ and $\Sigma_{2}$ are empty. When $N-\Lambda<$ $m \leq N+\Lambda, \Sigma_{1}=\emptyset$. Finally, for $m>N+\Lambda$, the four sets have at least one element.

Let us first consider the case $m>N+\Lambda$. We now give an upper bound of $\Delta_{l}^{(m)}$ on each of the four sets. 
(i) For $l \in \Sigma_{1}$, we have, thanks to (37) and (13),

$$
\left|\Delta_{l}^{(m)}\right| \leq M_{H} \sqrt{\frac{2}{\pi l}}\left(\frac{2 l}{\mathrm{e}|k| \mathbf{z}_{i}-\mathbf{z}_{j} \mid}\right)^{l} \frac{M_{J}}{\sqrt{2 \pi(m-l)}}\left(\frac{\mathrm{e}|k|\left|\mathbf{x}^{\prime}-\mathbf{z}_{j}\right|}{2(m-l)}\right)^{m-l} .
$$

We apply Proposition 12 part (ii) to $x=l, y=m-\Lambda$ and $a=$ $\frac{\mathrm{e}}{2}|k| \mathbf{z}_{i}-\mathbf{z}_{j} \mid$. Since $N<l \leq m-\Lambda$, we have $y \geq x>N>\Lambda \geq$ $\frac{\mathrm{e}}{2}|k| \mathbf{z}_{i}-\mathbf{z}_{j} \mid=a$, which implies that

$$
\frac{1}{\sqrt{l}}\left(\frac{2 l}{\mathrm{e}|k| \mathbf{z}_{i}-\mathbf{z}_{j} \mid}\right)^{l} \leq \frac{1}{\sqrt{N}}\left(\frac{2(m-\Lambda)}{\mathrm{e}|k| \mathbf{z}_{i}-\mathbf{z}_{j} \mid}\right)^{m-\Lambda}
$$

Since $\Lambda \leq m-l \leq m-N$ and by the definition of $\Lambda$, we get

$$
\frac{\mathrm{e}|k| \mathbf{x}^{\prime}-\mathbf{z}_{j} \mid}{2(m-l)} \leq \frac{\mathrm{e}|k|\left|\mathbf{x}^{\prime}-\mathbf{z}_{j}\right|}{2 \Lambda} \leq \frac{\mathrm{e}|k| \max _{j} \max _{\mathbf{x}^{\prime} \in A_{j}}\left|\mathbf{x}^{\prime}-\mathbf{z}_{j}\right|}{2 \Lambda} \leq 1
$$

Therefore

$$
\frac{1}{\sqrt{m-l}}\left(\frac{\mathrm{e}|k|\left|\mathbf{x}^{\prime}-\mathbf{z}_{j}\right|}{2(m-l)}\right)^{m-l} \leq \frac{1}{\sqrt{\Lambda}} .
$$

Since the set $\Sigma_{1}$ has $m-\Lambda-N$ terms, we arrive at

$$
\sum_{l \in \Sigma_{1}}\left|\Delta_{l}^{(m)}\right| \leq \frac{M_{J} M_{H}}{\pi \sqrt{\Lambda}} \frac{m-\Lambda-N}{\sqrt{N}}\left(\frac{2(m-\Lambda)}{\mathrm{e}|k| \mathbf{z}_{i}-\mathbf{z}_{j} \mid}\right)^{m-\Lambda} .
$$

(ii) For $l \in \Sigma_{2}$, the relation (42) is used to bound $\tilde{\beta}_{m-l}$ uniformly $\left|\tilde{\beta}_{m-l}\right| \leq$ $C_{\tilde{\beta}}$. The constant $C_{\tilde{\beta}}$ depends only on $\Lambda$ and $R$. Thus

$$
\left|\Delta_{l}^{(m)}\right| \leq C_{\tilde{\beta}} M_{H} \sqrt{\frac{2}{\pi l}}\left(\frac{2 l}{\mathrm{e}|k| \mathbf{z}_{i}-\mathbf{z}_{j} \mid}\right)^{l} .
$$

As in the previous item, one can show that the greatest term of $\left(\frac{2 l}{\mathrm{e} k\left|\mathbf{z}_{i}-\mathbf{z}_{j}\right|}\right)^{l}$ for $l \in \Sigma_{2}$ is obtained at $l=m+\Lambda$. Since $\Sigma_{2}$ is composed of $2 \Lambda$ terms, one may write

$$
\sum_{l \in \Sigma_{2}}\left|\Delta_{l}^{(m)}\right| \leq 2 \Lambda C_{\tilde{\beta}} M_{H} \sqrt{\frac{2}{\pi(m-\Lambda)}}\left(\frac{2(m+\Lambda)}{\mathrm{e}|k| \mathbf{z}_{i}-\mathbf{z}_{j} \mid}\right)^{m+\Lambda} .
$$

(iii) Let us consider the case when $l \in \Sigma_{3}$. Since $l>m+\Lambda$, (14) implies that

$$
\sum_{l \in \Sigma_{3}}\left|\Delta_{l}^{(m)}\right| \leq \frac{M_{J} M_{H}}{\pi}\left(\frac{2}{|k|\left|\mathbf{x}^{\prime}-\mathbf{z}_{j}\right|}\right)^{m} \sum_{l=m+\Lambda}^{\infty} l^{m}\left(\frac{\left|\mathbf{x}^{\prime}-\mathbf{z}_{j}\right|}{\left|\mathbf{z}_{i}-\mathbf{z}_{j}\right|}\right)^{l} .
$$

By Proposition 13 with $M=m, L=m+\Lambda$ and $x=\frac{\left|\mathbf{x}^{\prime}-\mathbf{z}_{j}\right|}{\left|\mathbf{z}_{i}-\mathbf{z}_{j}\right|}<\frac{1}{\sqrt{\mathrm{e}}}$, we get $\sum_{l \in \Sigma_{3}}\left|\Delta_{l}^{(m)}\right| \leq \frac{C_{g} M_{J} M_{H}}{\pi}\left(\frac{2}{|k|\left|\mathbf{x}^{\prime}-\mathbf{z}_{j}\right|}\right)^{m}(2(m+\Lambda))^{m} \frac{\left(\frac{\left|\mathbf{x}^{\prime}-\mathbf{z}_{j}\right|}{\mathbf{z}_{i}-\mathbf{z}_{j} \mid}\right)^{m+\Lambda}}{\left(1-\frac{\left|\mathbf{x}^{\prime}-\mathbf{z}_{j}\right|}{\left|\mathbf{z}_{i}-\mathbf{z}_{j}\right|}\right)^{m+1}}$. 
(iv) We want to bound the $\operatorname{sum} \sum_{l \in \Sigma_{4}}\left|\Delta_{l}^{(m)}\right|$ by $\sum_{l \in \Sigma_{3}}\left|\Delta_{l}^{(m)}\right|$ for any $m \in \mathbb{N}$. To this end, we define a function $\mathcal{L}_{m}$ which maps $\Sigma_{4}$ onto $\Sigma_{3}$. The smallest element of the set $\Sigma_{3}$ is equal to $m+\Lambda$ when $m+\Lambda>N$ and is equal to $N$ when $m+\Lambda \leq N$. The mapping $\mathcal{L}_{m}$ is thus defined as follows:

$$
\begin{aligned}
\left.\mathcal{L}_{m}: \Sigma_{4}=\right]-\infty,-N[\cap \mathbb{Z} & \left.\rightarrow \Sigma_{3}=\right] m+\Lambda, \infty[\cap \mathbb{Z} \text { if } m+\Lambda>N \\
l & \mapsto m+\Lambda-N+|l|
\end{aligned}
$$

and

$\left.\mathcal{L}_{m}: \Sigma_{4}=\right]-\infty,-N\left[\cap \mathbb{Z} \rightarrow \Sigma_{3}=\right] N, \infty[\cap \mathbb{Z}$ if $m+\Lambda \leq N$.

$$
l \mapsto|l|
$$

When $m+\Lambda>N$, we apply Proposition 12 part (i) to $x=|l|+m$, $y=|l|-N+\Lambda$ and $a=\frac{\mathrm{e}}{2}|k|\left|\mathbf{x}^{\prime}-\mathbf{z}_{j}\right|$. For $l \in \Sigma_{4}$, we have $x \geq y \geq \Lambda \geq a$, so that by (13)

$$
\begin{aligned}
\left|\tilde{\beta}_{m-l}\right| & \leq \frac{M_{J}}{\sqrt{2 \pi(l l \mid+m)}}\left(\frac{\mathrm{e}|k| \mathbf{x}^{\prime}-\mathbf{z}_{j} \mid}{2(l \mid+m)}\right)^{|l|+m} \\
& \leq \frac{M_{J}}{\sqrt{2 \pi(l \mid-N+\Lambda)}}\left(\frac{\mathrm{e}|k|\left|\mathbf{x}^{\prime}-\mathbf{z}_{j}\right|}{2(l \mid-N+\Lambda)}\right)^{|l|-N+\Lambda} \\
& =\frac{M_{J}}{\sqrt{2 \pi\left(\mathcal{L}_{m}(l)-m\right)}}\left(\frac{\mathrm{e}|k|\left|\mathbf{x}^{\prime}-\mathbf{z}_{j}\right|}{2\left(\mathcal{L}_{m}(l)-m\right)}\right)^{\mathcal{L}_{m}(l)-m} .
\end{aligned}
$$

Hence by (36)

$$
\left|\tilde{\beta}_{m-l}\right| \leq \frac{M_{J}}{m_{J}}\left|\tilde{\beta}_{\mathcal{L}_{m}(l)-m}\right| .
$$

Now if $m+\Lambda \leq N$, we directly have

$$
\left|\tilde{\beta}_{m-l}\right| \leq \frac{M_{J}}{\sqrt{2 \pi(l \mid-m)}}\left(\frac{\mathrm{e}|k| \mathbf{x}^{\prime}-\mathbf{z}_{j} \mid}{2(l \mid-m)}\right)^{|l|-m} \leq \frac{M_{J}}{m_{J}}\left|\tilde{\beta}_{\mathcal{L}_{m}(l)-m}\right| .
$$

Let us now consider $H_{l}^{(1)}\left(k\left|\mathbf{z}_{i}-\mathbf{z}_{j}\right|\right)$. To bound this term, we apply Proposition 12 part (ii) to $x=|l|, y=\mathcal{L}_{m}(l)$ and $a=\frac{\mathrm{e}}{2}|k| \mathbf{z}_{i}-\mathbf{z}_{j} \mid$. For $l \in \Sigma_{4}$ and in both cases $m+\Lambda>N$ and $m+\Lambda \leq N$, we have $y \geq x \geq N \geq a$, so that with (37)

$$
\begin{aligned}
\left|H_{l}^{(1)}\left(k \mathbf{z}_{i}-\mathbf{z}_{j} \mid\right)\right| & \leq M_{H} \sqrt{\frac{2}{\pi|l|}}\left(\frac{2|l|}{\mathrm{e}|k| \mathbf{z}_{i}-\mathbf{z}_{j} \mid}\right)^{|l|} \\
& \leq M_{H} \sqrt{\frac{\mathcal{L}_{m}(l)}{|l|}} \sqrt{\frac{2}{\pi \mathcal{L}_{m}(l)}}\left(\frac{2 \mathcal{L}_{m}(l)}{\mathrm{e}|k| \mathbf{z}_{i}-\mathbf{z}_{j} \mid}\right)^{\mathcal{L}_{m}(l)} \\
& \leq \frac{M_{H}}{m_{H}} \sqrt{\frac{\mathcal{L}_{m}(l)}{|l|}}\left|H_{\mathcal{L}_{m}(l)}^{(1)}\left(k\left|\mathbf{z}_{i}-\mathbf{z}_{j}\right|\right)\right|
\end{aligned}
$$

When $m+\Lambda \leq N, \mathcal{L}_{m}(l)=|l|$. When $m+\Lambda>N$, we have

$$
\frac{\mathcal{L}_{m}(l)}{|l|}=\frac{m+\Lambda-N+|l|}{|l|} \leq \frac{m+\Lambda}{|l|} \leq \frac{m+\Lambda}{N} .
$$


Therefore, in both cases $m>N-\Lambda$ and $m \leq N-\Lambda$, one may write $\frac{\mathcal{L}_{m}(l)}{|l|} \leq \max \left(1, \frac{m+\Lambda}{N}\right)$, and thus

$$
\begin{aligned}
\left|\Delta_{l}^{(m)}\right| & =\left|\tilde{\beta}_{m-l}\right|\left|H_{l}^{(1)}\left(k \mathbf{z}_{i}-\mathbf{z}_{j} \mid\right)\right| \\
& \leq \frac{M_{J} M_{H}}{m_{J} m_{H}} \max \left(1, \sqrt{\frac{m+\Lambda}{N}}\right)\left|\tilde{\beta}_{m-\mathcal{L}_{m}(l)}\right|\left|H_{\mathcal{L}_{m}(l)}^{(1)}\left(k \mathbf{z}_{i}-\mathbf{z}_{j} \mid\right)\right| \\
& =\frac{M_{J} M_{H}}{m_{J} m_{H}} \max \left(1, \sqrt{\frac{m+\Lambda}{N}}\right)\left|\Delta_{\mathcal{L}_{m}(l)}^{(m)}\right|
\end{aligned}
$$

Finally

$$
\begin{aligned}
\sum_{l \in \Sigma_{4}}\left|\Delta_{l}^{(m)}\right| & \leq \frac{M_{J} M_{H}}{m_{J} m_{H}} \max \left(1, \sqrt{\frac{m+\Lambda}{N}}\right) \sum_{l \in \Sigma_{4}}\left|\Delta_{\mathcal{L}_{m}(l)}^{(m)}\right| \\
& \leq \frac{M_{J} M_{H}}{m_{J} m_{H}} \max \left(1, \sqrt{\frac{m+\Lambda}{N}}\right) \sum_{l \in \Sigma_{3}}\left|\Delta_{l}^{(m)}\right| .
\end{aligned}
$$

At this point, we have a bound of the sums $\sum_{l}\left|\Delta_{l}^{(m)}\right|$ over the four sets $\Sigma_{1}$, $\Sigma_{2}, \Sigma_{3}$ and $\Sigma_{4}$. Let us use them to bound $\left|\gamma_{m}-\gamma_{m}^{(N)}\right|$. The leading behavior of the bounds of the sums on $\Sigma_{1}, \Sigma_{2}$ are nearly the same. More precisely, the sum on $\Sigma_{2}$ is greater than that on $\Sigma_{1}$, i.e.

$$
\sum_{l \in \Sigma_{1}}\left|\Delta_{l}^{(m)}\right| \leq \sum_{l \in \Sigma_{2}}\left|\Delta_{l}^{(m)}\right| .
$$

Let us first consider the case when $m>N+\Lambda$. The truncation error becomes

$$
\begin{aligned}
& \left|\gamma_{m}-\gamma_{m}^{(N)}\right| \leq 2 \sum_{l \in \Sigma_{2}}\left|\Delta_{l}^{(m)}\right|+\left(1+\frac{M_{J} M_{H}}{m_{J} m_{H}} \max \left(1, \sqrt{\frac{m+\Lambda}{N}}\right)\right) \sum_{l \in \Sigma_{3}}\left|\Delta_{l}^{(m)}\right| \\
& \leq 4 \Lambda C_{\tilde{\beta}} M_{H} \sqrt{\frac{2}{\pi(m-\Lambda)}\left(\frac{2(m+\Lambda)}{\mathrm{e}|k| \mathbf{z}_{i}-\mathbf{z}_{j} \mid}\right)^{m+\Lambda}} \\
& \quad+\left(1+\frac{M_{J} M_{H}}{m_{J} m_{H}}\right) \sqrt{\frac{m+\Lambda}{N}} \frac{C_{g} M_{J} M_{H}}{\pi}\left(\frac{4(m+\Lambda)}{\mathrm{e}|k|\left|\mathbf{x}^{\prime}-\mathbf{z}_{j}\right|}\right)^{m} \mathrm{e}^{m} \frac{\left(\frac{\left|\mathbf{x}^{\prime}-\mathbf{z}_{j}\right|}{\left|\mathbf{z}_{i}-\mathbf{z}_{j}\right|}\right)^{m+\Lambda}}{\left(1-\frac{\left|\mathbf{x}^{\prime}-\mathbf{z}_{j}\right|}{\left|\mathbf{z}_{i}-\mathbf{z}_{j}\right|}\right)^{m+1}} .
\end{aligned}
$$

Since $m>N+\Lambda \geq \Lambda$, we get $\sqrt{\frac{m+\Lambda}{N}} \leq \sqrt{\frac{2 m}{N}}$. Then, the relation $(m+\Lambda)^{m} \leq$ $\mathrm{e}^{\Lambda} m^{m}$ yields

$$
\begin{aligned}
\left|\gamma_{m}-\gamma_{m}^{(N)}\right| \leq 4 \Lambda C_{\tilde{\beta}} M_{H} \sqrt{\frac{2}{\pi(m-\Lambda)}}\left(\frac{2(m+\Lambda)}{\mathrm{e}|k|\left|\mathbf{z}_{i}-\mathbf{z}_{j}\right|}\right)^{m+\Lambda} \\
\quad+\left(1+\frac{M_{J} M_{H}}{m_{J} m_{H}}\right) \frac{C_{g} M_{J} M_{H}}{\pi} \sqrt{\frac{2 m}{N}}\left(\frac{4 m}{\mathrm{e}|k|\left|\mathbf{x}^{\prime}-\mathbf{z}_{j}\right|}\right)^{m} \frac{\left(\mathrm{e} \frac{\left|\mathbf{x}^{\prime}-\mathbf{z}_{j}\right|}{\mathbf{z}_{i}-\mathbf{z}_{j} \mid}\right)^{m+\Lambda}}{\left(1-\frac{\left|\mathbf{x}^{\prime}-\mathbf{z}_{j}\right|}{\left|\mathbf{z}_{i}-\mathbf{z}_{j}\right|}\right)^{m+1}} .
\end{aligned}
$$


Thus, when $m>N+\Lambda,(12)$ holds for some constants $C_{1}^{(+)}$and $C_{2}^{(+)}$which can be chosen independent of $m$ and $N$.

When $N-\Lambda<m \leq N+\Lambda$, the set $\Sigma_{1}$ is empty and the sum on $\Sigma_{2}$ is not "full" in the sense that it is only composed of $m+\Lambda-N$ terms instead of $2 \Lambda$ as in the previous case. More precisely, $\Sigma_{2}=\{l \in \mathbb{Z}, N<l \leq m+\Lambda\}$. Since $m+\Lambda-N \leq 2 \Lambda$, the same bound is attained

$$
\sum_{l=N+1}^{m+\Lambda}\left|\Delta_{l}^{(m)}\right| \leq 2 \Lambda C_{\tilde{\beta}} M_{H} \sqrt{\frac{2}{\pi(m-\Lambda)}}\left(\frac{2(m+\Lambda)}{\mathrm{e}|k| \mathbf{z}_{i}-\mathbf{z}_{j} \mid}\right)^{m+\Lambda} .
$$

Thus, since $\sqrt{\frac{m+\Lambda}{N}} \leq \sqrt{1+\frac{2 \Lambda}{N}} \leq \sqrt{3},(12)$ is also satisfied for $N-\Lambda<m \leq$ $N+\Lambda$, i.e.

$$
\begin{aligned}
\left|\gamma_{m}-\gamma_{m}^{(N)}\right| \leq & 2 \Lambda C_{\tilde{\beta}} M_{H} \sqrt{\frac{2}{\pi(m-\Lambda)}}\left(\frac{2(m+\Lambda)}{\mathrm{e}|k| \mathbf{z}_{i}-\mathbf{z}_{j} \mid}\right)^{m+\Lambda} \\
& +\sqrt{3}\left(1+\frac{M_{J} M_{H}}{m_{J} m_{H}}\right) \frac{C_{g} M_{J} M_{H}}{\pi} \\
& \cdot\left(\frac{4 m}{\mathrm{e}|k|\left|\mathbf{x}^{\prime}-\mathbf{z}_{j}\right|}\right)^{m} \frac{\left(\mathrm{e} \frac{\left|\mathbf{x}^{\prime}-\mathbf{z}_{j}\right|}{\mathbf{z}_{i}-\mathbf{z}_{j} \mid}\right)^{m+\Lambda}}{\left(1-\frac{\left|\mathbf{x}^{\prime}-\mathbf{z}_{j}\right|}{\mathbf{z}_{i}-\mathbf{z}_{j} \mid}\right)^{m+1}} .
\end{aligned}
$$

Finally for $0 \leq m \leq N-\Lambda$, the sum on $\Sigma_{3}$ can be bounded as in part (iii) of this proof (actually we only have to replace $L=m+\Lambda$ by $N$ when using Proposition 13)

$$
\frac{m_{J} m_{H}}{M_{J} M_{H}} \sum_{l \in \Sigma_{4}}\left|\Delta_{l}^{(m)}\right| \leq \sum_{l \in \Sigma_{3}}\left|\Delta_{l}^{(m)}\right| \leq \frac{C_{g} M_{J} M_{H}}{\pi}\left(\frac{4 N}{|k|\left|\mathbf{x}^{\prime}-\mathbf{z}_{j}\right|}\right)^{m} \frac{\left(\frac{\left|\mathbf{x}^{\prime}-\mathbf{z}_{j}\right|}{\mathbf{z}_{i}-\mathbf{z}_{j} \mid}\right)^{N}}{\left(1-\frac{\left|\mathbf{x}^{\prime}-\mathbf{z}_{j}\right|}{\left|\mathbf{z}_{i}-\mathbf{z}_{j}\right|}\right)^{m+1}} .
$$

Therefore when $0 \leq m \leq N-\Lambda$, we have

$$
\left|\gamma_{m}-\gamma_{m}^{(N)}\right| \leq\left(1+\frac{M_{J} M_{H}}{m_{J} m_{H}}\right) \frac{C_{g} M_{J} M_{H}}{\pi}\left(\frac{4 N}{|k|\left|\mathbf{x}^{\prime}-\mathbf{z}_{j}\right|}\right)^{m} \frac{\left(\frac{\left|\mathbf{x}^{\prime}-\mathbf{z}_{j}\right|}{\mathbf{z}_{i}-\mathbf{z}_{j} \mid}\right)^{N}}{\left(1-\frac{\left|\mathbf{x}^{\prime}-\mathbf{z}_{j}\right|}{\left|\mathbf{z}_{i}-\mathbf{z}_{j}\right|}\right)^{m+1}} .
$$

which proves (11) for some constant $C^{(-)}$.

In (11) and (12), the term $\left|\mathbf{x}^{\prime}-\mathbf{z}_{j}\right|$ can be removed from the denominators, so that (11) and (12) hold for all $\mathbf{x} \in D_{\mathbf{z}_{i}}$ and $\mathbf{x}^{\prime} \in D_{\mathbf{z}_{j}}$ (even for $\mathbf{x}^{\prime}=\mathbf{z}_{j}$ ).

The following theorem plays a central role in this paper since it proves the convergence of the FMM.

Theorem 2. $\quad$ Let $\Lambda$ be the smallest integer greater than the following four numbers

$$
\begin{array}{cl}
C|k|^{2} \max _{i, j}\left|\mathbf{z}_{i}-\mathbf{z}_{j}\right|^{2}, & \frac{\mathrm{e}}{2}|k| \max _{i, j}\left|\mathbf{z}_{i}-\mathbf{z}_{j}\right|, \\
C|k|^{2} \max _{i} \max _{\mathbf{x} \in A_{i}}\left|\mathbf{x}-\mathbf{z}_{i}\right|^{2}, & \frac{\mathrm{e}}{2}|k| \max _{i} \max _{\mathbf{x} \in A_{i}}\left|\mathbf{x}-\mathbf{z}_{i}\right|,
\end{array}
$$


where $C$ is given in Proposition 8. Let $A_{i}$ and $A_{j}$ be two different aggregates. We consider $\mathbf{x} \in A_{i}$ and $\mathbf{x}^{\prime} \in A_{j}$ such that

$$
\frac{\left|\mathbf{x}-\mathbf{z}_{i}\right|}{\left|\mathbf{z}_{i}-\mathbf{z}_{j}\right|}<1, \quad \mathrm{e} \frac{\left|\mathbf{x}^{\prime}-\mathbf{z}_{j}\right|}{\left|\mathbf{z}_{i}-\mathbf{z}_{j}\right|}<1, \quad \frac{\left|\mathbf{x}^{\prime}-\mathbf{z}_{j}\right|}{\left|\mathbf{z}_{i}-\mathbf{z}_{j}\right|}+2 \mathrm{e} \frac{\left|\mathbf{x}-\mathbf{z}_{i}\right|}{\left|\mathbf{z}_{i}-\mathbf{z}_{j}\right|}<1 .
$$

Then for all $N>2 \Lambda$, we have that

$$
\begin{aligned}
\left|G\left(\mathbf{x}, \mathbf{x}^{\prime}\right)-G_{N}^{\mathcal{C}}\left(\mathbf{x}, \mathbf{x}^{\prime}\right)\right| \leq C_{1} N^{\Lambda}\left(\mathrm{e} \frac{\left|\mathbf{x}^{\prime}-\mathbf{z}_{j}\right|}{\left|\mathbf{z}_{i}-\mathbf{z}_{j}\right|}\right)^{N-\Lambda}+C_{2} N^{\Lambda}\left(\frac{\left|\mathbf{x}-\mathbf{z}_{i}\right|}{\left|\mathbf{z}_{i}-\mathbf{z}_{j}\right|}\right)^{N-\Lambda} \\
+C_{3} N\left(\frac{2 \mathrm{e} \frac{\left|\mathbf{x}-\mathbf{z}_{i}\right|}{\left|\mathbf{z}_{i}-\mathbf{z}_{j}\right|}}{1-\frac{\left|\mathbf{x}^{\prime}-\mathbf{z}_{j}\right|}{\left|\mathbf{z}_{i}-\mathbf{z}_{j}\right|}}\right)^{N-\Lambda}
\end{aligned}
$$

where $C_{1}, C_{2}$ and $C_{3}$ are independent of $N$. In particular, $G_{N}^{\mathcal{C}}\left(\mathbf{x}, \mathbf{x}^{\prime}\right)$ converges uniformly to $G\left(\mathbf{x}, \mathbf{x}^{\prime}\right)$ under the condition (16). We can give another condition which is only sufficient, but which is symmetric in $i$ and $j$ :

$$
\left\{\begin{array}{l}
\frac{\left|\mathbf{x}^{\prime}-\mathbf{z}_{j}\right|}{\mathbf{z}_{i}-\mathbf{z}_{j} \mid}<\frac{1}{1+2 \mathrm{e}} \\
\frac{\left|\mathbf{x}-\mathbf{z}_{i}\right|}{\left|\mathbf{z}_{i}-\mathbf{z}_{j}\right|}<\frac{1}{1+2 \mathrm{e}}
\end{array}\right.
$$

Proof. We only have to show (17). The rest of the theorem is straightforward. The error of the FMM is

$$
\left|G\left(\mathbf{x}, \mathbf{x}^{\prime}\right)-G_{N}^{\mathcal{C}}\left(\mathbf{x}, \mathbf{x}^{\prime}\right)\right| \leq \frac{1}{4} \sum_{m \in \mathbb{Z}}\left|\gamma_{m}-\gamma_{m}^{(N)}\right|\left|J_{m}\left(|k|\left|\mathbf{x}-\mathbf{z}_{i}\right|\right)\right| .
$$

With the help of Lemma 1, the sum in the right hand side is split into two terms

$$
\left|G\left(\mathbf{x}, \mathbf{x}^{\prime}\right)-G_{N}^{\mathcal{C}}\left(\mathbf{x}, \mathbf{x}^{\prime}\right)\right| \leq \frac{S^{(-)}}{4}+\frac{S^{(+)}}{4}
$$

where by Lemma 1

$$
\begin{aligned}
S^{(-)} & =\sum_{|m| \leq N-\Lambda}\left|\gamma_{m}-\gamma_{m}^{(N)}\right|\left|J_{m}\left(|k|\left|\mathbf{x}-\mathbf{z}_{i}\right|\right)\right| \\
& \leq \sum_{|m| \leq N-\Lambda} C^{(-)}\left(\frac{4 N}{|k|\left|\mathbf{x}^{\prime}-\mathbf{z}_{j}\right|}\right)^{|m|} \frac{\left(\frac{\left|\mathbf{x}^{\prime}-\mathbf{z}_{j}\right|}{\mathbf{z}_{i}-\mathbf{z}_{j} \mid}\right)^{N}}{\left(1-\frac{\left|\mathbf{x}^{\prime}-\mathbf{z}_{j}\right|}{\left|\mathbf{z}_{i}-\mathbf{z}_{j}\right|}\right)^{|m|}}\left|J_{m}\left(|k|\left|\mathbf{x}-\mathbf{z}_{i}\right|\right)\right|
\end{aligned}
$$

and

$$
\begin{aligned}
S^{(+)} & =\sum_{|m|>N-\Lambda}\left|\gamma_{m}-\gamma_{m}^{(N)}\right|\left|J_{m}\left(|k|\left|\mathbf{x}-\mathbf{z}_{i}\right|\right)\right| \\
\leq & \sum_{|m|>N-\Lambda} C_{1}^{(+)}\left(\frac{2(|m|+\Lambda)}{\mathrm{e}|k|\left|\mathbf{z}_{i}-\mathbf{z}_{j}\right|}\right)^{|m|+\Lambda}\left|J_{m}\left(|k|\left|\mathbf{x}-\mathbf{z}_{i}\right|\right)\right| \\
& +\sum_{|m|>N-\Lambda} C_{2}^{(+)} \sqrt{m}\left(\frac{4|m|}{\mathrm{e}|k|\left|\mathbf{x}^{\prime}-\mathbf{z}_{j}\right|}\right)^{|m|} \frac{\left(\mathrm{e} \frac{\left|\mathbf{x}^{\prime}-\mathbf{z}_{j}\right|}{\left|\mathbf{z}_{i}-\mathbf{z}_{j}\right|}\right)^{|m|+\Lambda}}{\left(1-\frac{\left|\mathbf{x}^{\prime}-\mathbf{z}_{j}\right|}{\left|\mathbf{z}_{i}-\mathbf{z}_{j}\right|}\right)^{|m|}}\left|J_{m}\left(|k|\left|\mathbf{x}-\mathbf{z}_{i}\right|\right)\right|
\end{aligned}
$$


- Let us begin with $S^{(-)}$. By (36), we split $S^{(-)}$into two sums, one when (36) cannot be used (namely for $0 \leq m \leq \Lambda$ ), and the other when (36) can be used (namely for $\Lambda+1 \leq m \leq N-\Lambda$ ). By symmetry, only positive values of $m$ have to be considered. Thus

$$
\begin{aligned}
S^{(-)} \leq & 2 C^{(-)}\left(\frac{\left|\mathbf{x}^{\prime}-\mathbf{z}_{j}\right|}{\left|\mathbf{z}_{i}-\mathbf{z}_{j}\right|}\right)^{N} \sum_{m=0}^{\Lambda}\left(\frac{4 N}{|k|\left|\mathbf{x}^{\prime}-\mathbf{z}_{j}\right|}\right)^{m} \frac{\left|J_{m}\left(|k|\left|\mathbf{x}-\mathbf{z}_{i}\right|\right)\right|}{\left(1-\frac{\left|\mathbf{x}^{\prime}-\mathbf{z}_{j}\right|}{\left|\mathbf{z}_{i}-\mathbf{z}_{j}\right|}\right)^{m}} \\
& +2 C^{(-)} M_{J}\left(\frac{\left|\mathbf{x}^{\prime}-\mathbf{z}_{j}\right|}{\left|\mathbf{z}_{i}-\mathbf{z}_{j}\right|}\right)^{N} \sum_{m=\Lambda+1}^{N-\Lambda} \frac{\left(\frac{2 \mathrm{e} N}{m}\right)^{m}}{\sqrt{2 \pi m}} \frac{\left(\frac{\left|\mathbf{x}-\mathbf{z}_{i}\right|}{\left|\mathbf{x}^{\prime}-\mathbf{z}_{j}\right|}\right)^{m}}{\left(1-\frac{\left|\mathbf{x}^{\prime}-\mathbf{z}_{j}\right|}{\left|\mathbf{z}_{i}-\mathbf{z}_{j}\right|}\right)^{m}} .
\end{aligned}
$$

For $0 \leq m \leq \Lambda$, the Bessel function satisfies $J_{m}\left(|k|\left|\mathbf{x}-\mathbf{z}_{i}\right|\right) \leq C_{\tilde{\beta}}$ by (42). Moreover, since $|k|\left|\mathbf{z}_{i}-\mathbf{z}_{j}\right| \leq \frac{2}{\mathrm{e}} \Lambda$, we have

$$
\frac{4 N}{|k|\left|\mathbf{x}^{\prime}-\mathbf{z}_{j}\right|\left(1-\frac{\left|\mathbf{x}^{\prime}-\mathbf{z}_{j}\right|}{\left|\mathbf{z}_{i}-\mathbf{z}_{j}\right|}\right)} \geq \frac{4 N}{|k|\left|\mathbf{x}^{\prime}-\mathbf{z}_{j}\right|} \geq \frac{2 \mathrm{e} N}{\Lambda} \geq 2 \mathrm{e}>1 .
$$

Hence, the sequence

$$
\left\{\left(\frac{4 N}{|k|\left|\mathbf{x}^{\prime}-\mathbf{z}_{j}\right|\left(1-\frac{\left|\mathbf{x}^{\prime}-\mathbf{z}_{j}\right|}{\mathbf{z}_{i}-\mathbf{z}_{j} \mid}\right)}\right)^{m}\right\}_{0 \leq m \leq \Lambda}
$$

is maximal when $m=\Lambda$.

In order to study the general term in the sum over $\Lambda+1 \leq m \leq N-\Lambda$, let us set

$$
y:=\frac{2 \mathrm{e} \frac{\left|\mathbf{x}-\mathbf{z}_{i}\right|}{\left|\mathbf{x}^{\prime}-\mathbf{z}_{j}\right|}}{1-\frac{\left|\mathbf{x}^{\prime}-\mathbf{z}_{j}\right|}{\left|\mathbf{z}_{i}-\mathbf{z}_{j}\right|}} .
$$

Then

$$
\begin{aligned}
S^{(-)} \leq & 2 C^{(-)}(\Lambda+1)\left(\frac{4 N}{|k|\left|\mathbf{x}^{\prime}-\mathbf{z}_{j}\right|}\right)^{\Lambda} \frac{C_{\tilde{\beta}}}{\left(1-\frac{\left|\mathbf{x}^{\prime}-\mathbf{z}_{j}\right|}{\left|\mathbf{z}_{i}-\mathbf{z}_{j}\right|}\right)}\left(\frac{\left|\mathbf{x}^{\prime}-\mathbf{z}_{j}\right|}{\left|\mathbf{z}_{i}-\mathbf{z}_{j}\right|}\right)^{N} \\
& +2 C^{(-)} M_{J}\left(\frac{\left|\mathbf{x}^{\prime}-\mathbf{z}_{j}\right|}{\left|\mathbf{z}_{i}-\mathbf{z}_{j}\right|}\right)^{N} \sum_{m=\Lambda+1}^{N-\Lambda} \frac{1}{\sqrt{2 \pi}}\left(y \frac{N}{m}\right)^{m} .
\end{aligned}
$$

Now we want to give an upper bound for the function $p(m)=\left(y \frac{N}{m}\right)^{m}$ for $\Lambda<m \leq N-\Lambda$. By setting $\gamma=\frac{m}{N}$, it is sufficient to find an upper bound for the function $q(\gamma)=\left(\frac{y}{\gamma}\right)^{\gamma}$ for $0<\gamma<1$, since $\max _{\Lambda<m \leq N-\Lambda} p(m) \leq$ $\left[\max _{\gamma \in] 0,1[} q(\gamma)\right]^{N}$. The maximum of $q(\gamma)$ is given by Proposition 12. For this, we must distinguish the following alternative:

(i) If $\frac{y}{\mathrm{e}} \leq 1$, then by Proposition 12 part (iv) with $a=y, x=\gamma$ and $A=1$, we get $\max _{\gamma \in] 0,1[} q(\gamma) \leq$ e. Hence $\sum_{m=\Lambda+1}^{N-\Lambda}\left(y \frac{N}{m}\right)^{m} \leq(N-2 \Lambda) \mathrm{e}^{N}$, so 
that

$$
\begin{aligned}
S^{(-)} \leq & 2 C^{(-)} C_{\tilde{\beta}}(\Lambda+1)\left(\frac{\frac{4}{|k| \mathbf{z}_{i}-\mathbf{z}_{j} \mid}}{1-\frac{\left|\mathbf{x}^{\prime}-\mathbf{z}_{j}\right|}{\left|\mathbf{z}_{i}-\mathbf{z}_{j}\right|}}\right)^{\Lambda} N^{\Lambda}\left(\frac{\left|\mathbf{x}^{\prime}-\mathbf{z}_{j}\right|}{\left|\mathbf{z}_{i}-\mathbf{z}_{j}\right|}\right)^{N-\Lambda} \\
& +\frac{2 C^{(-)} M_{J}}{\sqrt{2 \pi}}(N-2 \Lambda)\left(\mathrm{e} \frac{\left|\mathbf{x}^{\prime}-\mathbf{z}_{j}\right|}{\left|\mathbf{z}_{i}-\mathbf{z}_{j}\right|}\right)^{N} .
\end{aligned}
$$

(ii) If $\frac{y}{\mathrm{e}}>1$, then by Proposition 12 part (iii) with $a=y, x=\gamma$ and $A=1$, we get $\max _{\gamma \in] 0,1[} q(\gamma)=q(1)=y$. Thus $\sum_{m=\Lambda+1}^{N-\Lambda}\left(y \frac{N}{m}\right)^{m} \leq(N-2 \Lambda) y^{N}$, and

$$
\begin{aligned}
S^{(-)} \leq & 2 C^{(-)} C_{\tilde{\beta}}(\Lambda+1)\left(\frac{\frac{4}{|k| \mathbf{z}_{i}-\mathbf{z}_{j} \mid}}{1-\frac{\left|\mathbf{x}^{\prime}-\mathbf{z}_{j}\right|}{\left|\mathbf{z}_{i}-\mathbf{z}_{j}\right|}}\right)^{\Lambda} N^{\Lambda}\left(\frac{\left|\mathbf{x}^{\prime}-\mathbf{z}_{j}\right|}{\left|\mathbf{z}_{i}-\mathbf{z}_{j}\right|}\right)^{N-\Lambda} \\
& +\frac{2 C^{(-)} M_{J}(N-2 \Lambda)}{\sqrt{2 \pi}}\left(\frac{\left|\mathbf{x}^{\prime}-\mathbf{z}_{j}\right|}{\left|\mathbf{z}_{i}-\mathbf{z}_{j}\right|}\right)^{N}\left(\frac{2 \frac{\left|\mathbf{x}-\mathbf{z}_{i}\right|}{\mid \mathbf{x}^{\prime}-\mathbf{z}_{j} j}}{1-\frac{\left|\mathbf{x}^{\prime}-\mathbf{z}_{j}\right|}{\left|\mathbf{z}_{i}-\mathbf{z}_{j}\right|}}\right)^{N} .
\end{aligned}
$$

- The sum $S^{(+)}$is bounded in the same way. By (36)

$$
\begin{aligned}
& S^{(+)} \leq 2 C_{1}^{(+)} \sum_{m>N-\Lambda}\left(\frac{2(m+\Lambda)}{\mathrm{e}|k|\left|\mathbf{z}_{i}-\mathbf{z}_{j}\right|}\right)^{m+\Lambda} \frac{M_{J}}{\sqrt{2 \pi m}}\left(\frac{\mathrm{e}|k|\left|\mathbf{x}-\mathbf{z}_{i}\right|}{2 m}\right)^{m} \\
&+2 C_{2}^{(+)} \sum_{m>N-\Lambda} \sqrt{m}\left(\frac{4 m}{\mathrm{e}|k|\left|\mathbf{x}^{\prime}-\mathbf{z}_{j}\right|}\right)^{m} \frac{\left(\mathrm{e} \frac{\left|\mathbf{x}^{\prime}-\mathbf{z}_{j}\right|}{\mathbf{z}_{i}-\mathbf{z}_{j} \mid}\right)^{m+\Lambda}}{\left(1-\frac{\left|\mathbf{x}^{\prime}-\mathbf{z}_{j}\right|}{\mathbf{z}_{i}-\mathbf{z}_{j} \mid}\right)^{m}} \\
& \\
& \cdot \frac{M_{J}}{\sqrt{2 \pi m}}\left(\frac{\mathrm{e}|k|\left|\mathbf{x}-\mathbf{z}_{j}\right|}{2 m}\right)^{m} .
\end{aligned}
$$

Since $m>N-\Lambda>0$, we have $m \geq 1$. Hence by Proposition 11 part (iii)

$$
\frac{(m+\Lambda)^{m+\Lambda}}{m^{m}} \leq m^{\Lambda}(\Lambda+1)^{\Lambda+1},
$$

which proves that

$$
\begin{aligned}
S^{(+)} \leq & \frac{2 C_{1}^{(+)} M_{J}}{\sqrt{2 \pi}} \frac{(\Lambda+1)^{\Lambda+1}}{\mathrm{e}^{\Lambda}} \sum_{m>N-\Lambda}\left(\frac{2 m}{|k| \mathbf{z}_{i}-\mathbf{z}_{j} \mid}\right)^{\Lambda} \frac{1}{\sqrt{m}}\left(\frac{\left|\mathbf{x}-\mathbf{z}_{i}\right|}{\left|\mathbf{z}_{i}-\mathbf{z}_{j}\right|}\right)^{m} \\
& +\frac{2 C_{2}^{(+)} M_{J}}{\sqrt{2 \pi}}\left(\frac{\mathrm{e}\left|\mathbf{x}^{\prime}-\mathbf{z}_{j}\right|}{\left|\mathbf{z}_{i}-\mathbf{z}_{j}\right|}\right)^{\Lambda} \sum_{m>N-\Lambda}\left(\frac{2 \mathrm{e} \frac{\left|\mathbf{x}-\mathbf{z}_{i}\right|}{\mathbf{z}_{i}-\mathbf{z}_{j} \mid}}{1-\frac{\left|\mathbf{x}^{\prime}-\mathbf{z}_{j}\right|}{\left|\mathbf{z}_{i}-\mathbf{z}_{j}\right|}}\right)^{m}
\end{aligned}
$$


and by using Proposition 13 with $L=N-\Lambda, M=\Lambda$ and $x=\frac{\left|\mathbf{x}-\mathbf{z}_{i}\right|}{\left|\mathbf{z}_{i}-\mathbf{z}_{j}\right|}<\frac{1}{\sqrt{\mathrm{e}}}$, we attain to $\sum_{m>N-\Lambda} m^{\Lambda} x^{m} \leq C_{g}(2(N-\Lambda))^{\Lambda} \frac{x^{N-\Lambda}}{(1-x)^{\Lambda+1}}$. Therefore

$$
\begin{aligned}
& S^{(+)} \leq \frac{2 C_{1}^{(+)} M_{J} C_{g}}{\sqrt{2 \pi}} \frac{(\Lambda+1)^{\Lambda+1}}{\mathrm{e}^{\Lambda}}\left(\frac{2}{|k| \mathbf{z}_{i}-\mathbf{z}_{j} \mid}\right)^{\Lambda}(2(N-\Lambda))^{\Lambda} \frac{\left(\frac{\left|\mathbf{x}-\mathbf{z}_{i}\right|}{\mathbf{z}_{i}-\mathbf{z}_{j} \mid}\right)^{N-\Lambda}}{\left(1-\frac{\left|\mathbf{x}-\mathbf{z}_{i}\right|}{\left|\mathbf{z}_{i}-\mathbf{z}_{j}\right|}\right)^{\Lambda+1}}
\end{aligned}
$$

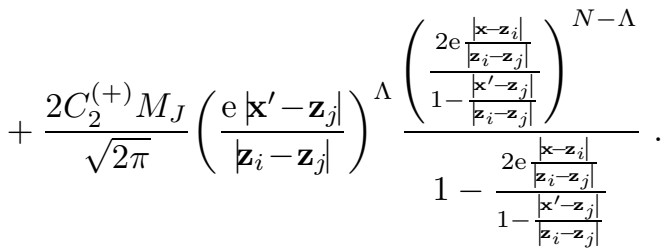

In putting together the upper bounds (19), (20) and (21), one automatically shows (17) for some constants $C_{1}, C_{2}$ and $C_{3}$ independent of $N$. We also notice that neither $\left|\mathbf{x}-\mathbf{z}_{i}\right|$ nor $\left|\mathbf{x}^{\prime}-\mathbf{z}_{j}\right|$ appear in a denominator of $C_{1}, C_{2}$ or $C_{3}$. Thus the cases $\mathbf{x}=\mathbf{z}_{i}$ and $\mathbf{x}^{\prime}=\mathbf{z}_{j}$ are also included.

Corollary 3. The expansions $\sum_{m \in \mathbb{Z}} \gamma_{m}^{(N)} J_{m}\left(k\left|\mathbf{x}-\mathbf{z}_{i}\right|\right)$ and $\sum_{m \in \mathbb{Z}} \gamma_{m} J_{m}\left(k\left|\mathbf{x}-\mathbf{z}_{i}\right|\right)$ converge uniformly, under the conditions of Theorem 2.

Proof. From Lemma 1, the expansion $\sum_{m \in \mathbb{Z}}\left|\gamma_{m}^{(N)}\right|$ converges. Combining this with (36), it is clear that $\sum_{m \in \mathbb{Z}} \gamma_{m}^{(N)} J_{m}\left(k\left|\mathbf{x}-\mathbf{z}_{i}\right|\right)$ converges uniformly.

On the other hand, from the proof of Theorem 2, the factor

$$
\sum_{m \in \mathbb{Z}}\left|\left(\gamma_{m}-\gamma_{m}^{(N)}\right) J_{m}\left(k\left|\mathbf{x}-\mathbf{z}_{i}\right|\right)\right|
$$

converges. Hence $\sum_{m \in \mathbb{Z}} \gamma_{m} J_{m}\left(k\left|\mathbf{x}-\mathbf{z}_{i}\right|\right)$ converges uniformly.

From Theorem 2, we see that the exact kernel cannot be replaced by an approximate one for all $\mathbf{x}$ and $\mathbf{x}^{\prime}$. Roughly speaking, convergence occurs when $\mathbf{x}$ is not too close to $\mathbf{x}^{\prime}$. So let us investigate what the condition (18) means exactly. We assume for the sake of simplicity and brevity that the boundary $\Gamma$ of the obstacle is locally planar (see Figure 3.a). We also assume that the length $D$ of the aggregates $A_{j}$ is the same for all $j$. Then $\left|\mathbf{x}-\mathbf{z}_{i}\right| \leq \frac{D}{2}$ for all $\mathbf{x} \in A_{i}$ and $\left|\mathbf{z}_{i}-\mathbf{z}_{i+4}\right|=4 D$. Hence $\frac{\left|\mathbf{x}-\mathbf{z}_{i}\right|}{\left|\mathbf{z}_{i}-\mathbf{z}_{i+4}\right|} \leq \frac{1}{8}<\frac{1}{1+2 \mathrm{e}}$ for all $\mathbf{x} \in A_{i}$. Thanks to Theorem 2, the approximate kernel converges to the exact one when $N \rightarrow \infty$. Therefore, the FMM approximation converges for all $\mathbf{x} \in A_{i}$ and $\mathbf{x}^{\prime} \in A_{j}$, when $A_{i}$ and $A_{j}$ satisfy $|i-j| \geq 4$.

This is again true when the curve $\Gamma$ has a mild curvature. For the circle (see Figure 3.b), if $D$ is now referred to as the angle which covers one whole aggregate $A_{j}$, then $\frac{\left|\mathbf{x}-\mathbf{z}_{i}\right|}{\left|\mathbf{z}_{i}-\mathbf{z}_{i+4}\right|} \leq \sqrt{\frac{1-\cos \left(\frac{D}{2}\right)}{1-\cos (4 D)}}$ for all $\mathbf{x} \in A_{i}$. This number is lower than $\frac{1}{1+2 \mathrm{e}}$ if $0<D \leq 32.2^{\underline{o}}$. This condition on the size of the aggregates is not restrictive at all.

Therefore, for a smooth obstacle, if the aggregate $A_{i}$ is fixed, then all except 7 aggregates $A_{j}$ generally satisfy the condition (18). For these 7 "nearby aggregates" the classical kernel must be used, and for the $p-7$ remaining aggregates (i.e. the "remote aggregates") the approximate kernel is used. 


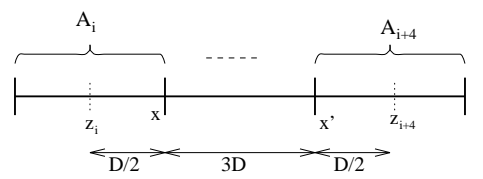

fig. a

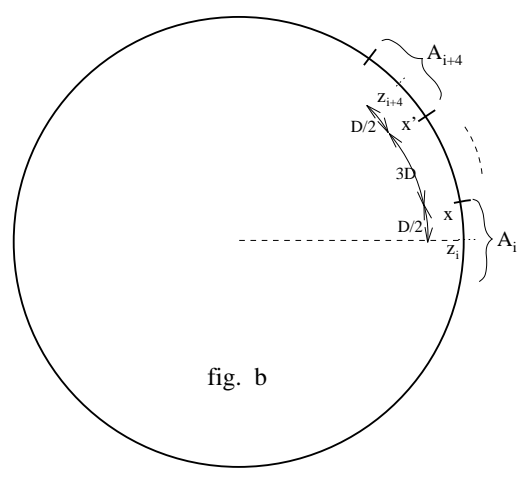

Figure 3. a: When $\Gamma$ is locally a straight line. b: When $\Gamma$ is a circle of radius 1 [then we have $\left|\mathbf{x}-\mathbf{z}_{i}\right|^{2} \leq 2\left(1-\cos \left(\frac{D}{2}\right)\right)$ and $\left.\left|\mathbf{z}_{i}-\mathbf{z}_{i+4}\right|^{2}=2(1-\cos (4 D))\right]$.

\section{Convergence analysis of the Discretized APPRoXimated Kernel}

Theorem 4. Let $\Lambda$ be the smallest integer greater than the following four numbers

$$
\begin{gathered}
C|k|^{2} \max _{i, j}\left|\mathbf{z}_{i}-\mathbf{z}_{j}\right|^{2}, \frac{\mathrm{e}}{2}|k| \max _{i, j}\left|\mathbf{z}_{i}-\mathbf{z}_{j}\right|, \\
C|k|^{2} \max _{i} \max _{\mathbf{x} \in A_{i}}\left|\mathbf{x}-\mathbf{z}_{i}\right|^{2}, \\
\frac{\mathrm{e}}{2}|k| \max _{i} \max _{\mathbf{x} \in A_{i}}\left|\mathbf{x}-\mathbf{z}_{i}\right|,
\end{gathered}
$$

where $C$ is given in Proposition 8. Let $A_{i}$ and $A_{j}$ be two different aggregates, and let us consider $\mathbf{x} \in A_{i}$ and $\mathbf{x}^{\prime} \in A_{j}$. Then for $N>\Lambda$, we have

$$
\begin{aligned}
\left|G_{N}^{\mathcal{C}}\left(\mathbf{x}, \mathbf{x}^{\prime}\right)-G_{N}^{\mathcal{D}}\left(\mathbf{x}, \mathbf{x}^{\prime}\right)\right| \leq & C_{4}\left(\frac{\mathrm{e}|k|\left(\left|\mathbf{x}-\mathbf{z}_{i}\right|+\left|\mathbf{x}^{\prime}-\mathbf{z}_{j}\right|\right)}{N}\right)^{N} \\
& +C_{5} \sqrt{N}\left(2 \frac{\left|\mathbf{x}-\mathbf{z}_{i}\right|+\left|\mathbf{x}^{\prime}-\mathbf{z}_{j}\right|}{\mathbf{z}_{i}-\mathbf{z}_{j} \mid}\right)^{N}
\end{aligned}
$$

where $C_{4}$ and $C_{5}$ are independent of $N$.

Combining this with Theorem $2, G_{N}^{\mathcal{D}}\left(\mathbf{x}, \mathbf{x}^{\prime}\right)$ converges uniformly to $G\left(\mathbf{x}, \mathbf{x}^{\prime}\right)$ under the condition (18).

Proof. Let us set $r=\left|\left(\mathbf{x}-\mathbf{z}_{i}\right)-\left(\mathbf{x}^{\prime}-\mathbf{z}_{j}\right)\right|, \alpha=\arg \left(\left(\mathbf{x}-\mathbf{z}_{i}\right)-\left(\mathbf{x}^{\prime}-\mathbf{z}_{j}\right)\right)$ and $z=\imath k r$. Then $\imath k\left(\left(\mathbf{x}-\mathbf{z}_{i}\right)-\left(\mathbf{x}^{\prime}-\mathbf{z}_{j}\right)\right) \cdot \mathbf{U}(\theta)=z \cos (\theta-\alpha)$. Hence by $(9)$

$$
G_{N}^{\mathcal{C}}\left(\mathbf{x}, \mathbf{x}^{\prime}\right)=\frac{1}{4 \pi^{2}} \int_{0}^{2 \pi} \exp (z \cos (\theta-\alpha)) \tau_{i j}^{(N)}(\theta) d \theta .
$$

Setting

$$
\mathcal{I}_{m}^{\mathcal{C}}:=\frac{1}{2 \pi} \int_{0}^{2 \pi} \exp (z \cos (\theta-\alpha)) \exp (\imath m \theta) d \theta,
$$

the continuous approximated kernel takes the form, thanks to (10),

$$
G_{N}^{\mathcal{C}}\left(\mathbf{x}, \mathbf{x}^{\prime}\right)=\frac{1}{2 \pi} \sum_{m=-N}^{N} \exp \left(-\imath m \arg \left(\mathbf{z}_{i}-\mathbf{z}_{j}\right)\right) K_{|m|}\left(-\imath k\left|\mathbf{z}_{i}-\mathbf{z}_{j}\right|\right) \mathcal{I}_{m}^{\mathcal{C}} .
$$


By introducing

$$
\mathcal{I}_{m}^{\mathcal{D}}:=\frac{1}{N_{T}} \sum_{n_{T}=1}^{N_{T}} \exp \left(z \cos \left(\frac{2 \pi n_{T}}{N_{T}}-\alpha\right)\right) \exp \left(\imath m \frac{2 \pi n_{T}}{N_{T}}\right),
$$

the discretized approximated kernel can also be written as

$$
G_{N}^{\mathcal{D}}\left(\mathbf{x}, \mathbf{x}^{\prime}\right)=\frac{1}{2 \pi} \sum_{m=-N}^{N} \exp \left(-\imath m \arg \left(\mathbf{z}_{i}-\mathbf{z}_{j}\right)\right) K_{|m|}\left(-\imath k\left|\mathbf{z}_{i}-\mathbf{z}_{j}\right|\right) \mathcal{I}_{m}^{\mathcal{D}} .
$$

Consequently

$$
\begin{aligned}
G_{N}^{\mathcal{C}}\left(\mathbf{x}, \mathbf{x}^{\prime}\right)-G_{N}^{\mathcal{D}}\left(\mathbf{x}, \mathbf{x}^{\prime}\right)=\frac{1}{2 \pi} \sum_{m=-N}^{N} \exp \left(-\imath m \arg \left(\mathbf{z}_{i}-\mathbf{z}_{j}\right)\right) \\
\cdot K_{|m|}\left(-\imath k \mathbf{z}_{i}-\mathbf{z}_{j} \mid\right)\left(\mathcal{I}_{m}^{\mathcal{C}}-\mathcal{I}_{m}^{\mathcal{D}}\right) .
\end{aligned}
$$

We notice that

$$
r=\left|\left(\mathbf{x}-\mathbf{z}_{i}\right)-\left(\mathbf{x}^{\prime}-\mathbf{z}_{j}\right)\right| \leq\left|\mathbf{x}-\mathbf{z}_{i}\right|+\left|\mathbf{x}^{\prime}-\mathbf{z}_{j}\right| .
$$

For reasons of symmetry, we only consider positive values of $m$.

- One may write

$$
\mathcal{I}_{m}^{\mathcal{C}}=\frac{1}{2 \pi} \int_{0}^{2 \pi} \sum_{l=0}^{\infty} \frac{z^{l} \cos ^{l}(\theta-\alpha)}{l !} \mathrm{e}^{\imath m \theta} d \theta=\sum_{l=0}^{\infty} \frac{z^{l}}{l !} I_{l}^{\mathcal{C}},
$$

where

$$
\begin{aligned}
I_{l}^{\mathcal{C}} & =\frac{1}{2 \pi} \int_{0}^{2 \pi} \cos ^{l}(\theta-\alpha) \mathrm{e}^{\imath m \theta} d \theta=\frac{1}{2 \pi} \int_{0}^{2 \pi}\left[\frac{\mathrm{e}^{\imath(\theta-\alpha)}+\mathrm{e}^{-\imath(\theta-\alpha)}}{2}\right]^{l} \mathrm{e}^{\imath m \theta} d \theta \\
& =\frac{1}{2 \pi} \sum_{l_{1}=0}^{l} \frac{C_{l}^{l_{1}}}{2^{l}} \exp \left(\imath\left(l-2 l_{1}\right) \alpha\right) \int_{0}^{2 \pi} \exp \left(\imath\left(m-l+2 l_{1}\right) \theta\right) d \theta
\end{aligned}
$$

where $C_{l}^{l_{1}}=\frac{l !}{l_{1} !\left(l-l_{1}\right) !}$. We remark that $\frac{1}{2 \pi} \int_{0}^{2 \pi} \mathrm{e}^{\imath m_{1}} d \theta$ is equal to 1 if $m_{1}=0$ and vanishes for all other integer values of $m_{1}$. Let us then consider the following three cases:

(i) $\underline{l<m}$ : Then $m-l+2 l_{1}$ never vanishes for $l_{1} \in\{0, \cdots, l\}$. Thus $I_{l}^{\mathcal{C}}=0$.

(ii) $l \geq m$ and $l-m$ is odd: There is no integer $l_{1}$ such that $2 l_{1}=l-m$. Hence $I_{l}^{\mathcal{C}}=0$.

(iii) $l \geq m$ and $l-m$ is even: Then $m-l+2 l_{1}$ vanishes if and only if $l_{1}=$ $\frac{l-m}{2} \in\{0, \cdots, l\}$. Hence $I_{l}^{\mathcal{C}}=\frac{C_{l}^{(l-m) / 2}}{2^{l}} \mathrm{e}^{\imath m \alpha}$.

Consequently

$$
\mathcal{I}_{m}^{\mathcal{C}}=\mathrm{e}^{\imath m \alpha} \sum_{l=m}^{\infty} \frac{z^{l}}{2^{l} l !} C_{l}^{(l-m) / 2}\left(\frac{1+(-1)^{l-m}}{2}\right) .
$$

We also conclude that when $l$ is odd $I_{l}^{\mathcal{C}}=0$, and otherwise $\left|I_{l}^{\mathcal{C}}\right| \leq \frac{C_{l}^{(l-m) / 2}}{2^{l}} \leq$ $\frac{\sum_{l_{1}=0}^{l} C_{l}^{l_{1}}}{2^{l}}=1$. Thus for all $l \in \mathbb{N}$

$$
\left|I_{l}^{\mathcal{C}}\right| \leq 1 .
$$


- Proceeding as in last step, one can show that

$$
\mathcal{I}_{m}^{\mathcal{D}}=\sum_{l=0}^{\infty} \frac{z^{l}}{l !} I_{l}^{\mathcal{D}}
$$

where

$$
I_{l}^{\mathcal{D}}=\sum_{l_{1}=0}^{l} \frac{C_{l}^{l_{1}}}{2^{l}} \exp \left(\imath\left(l-2 l_{1}\right) \alpha\right) S_{m-l+2 l_{1}},
$$

and $S_{m_{1}}:=\frac{1}{N_{T}} \sum_{n_{T}=1}^{N_{T}} \exp \left(\imath m_{1} \frac{2 \pi n_{T}}{N_{T}}\right)$. Let us then consider the four following cases

(i) $\underline{l<m}$ : Thanks to (5), we get $m-l+2 l_{1} \leq m+l<2 m \leq 2 N=N_{T}-1$. Moreover $m-l+2 l_{1}>2 l_{1} \geq 0$ for $l_{1} \in\{0, \cdots, l\}$. Hence by Proposition $14, I_{l}^{\mathcal{D}}=0$.

(ii) $l \geq m, l-m$ is odd and $l<N_{T}-m$ : We have $m-l+2 l_{1} \leq m+l<N_{T}$ and $m-l+2 l_{1} \geq m-l>2 m-N_{T} \geq-N_{T}$ (for $l_{1} \in\{0, \cdots, l\}$ ) since $m$ is supposed to be positive. Hence $\left|m-l+2 l_{1}\right| \leq N_{T}-1$. Moreover there is no integer $l_{1}$ such that $2 l_{1}=l-m$. Therefore by Proposition 14, $I_{l}^{\mathcal{D}}=0$.

(iii) $l \geq m, l-m$ is even and $l<N_{T}-m$ : As in the last step, $\left|m-l+2 l_{1}\right| \leq$ $N_{T}-1$. In addition, $m-l+2 l_{1}$ vanishes if and only if $l_{1}=\frac{l-m}{2} \in$ $\{0, \cdots, l\}$. Hence by Proposition 14, $I_{l}^{\mathcal{D}}=\frac{C_{l}^{(l-m) / 2}}{2^{l}} \mathrm{e}^{\imath m \alpha}$.

(iv) $l \geq m$, and $l \geq N_{T}-m$ : We cannot give an explicit value of $I_{l}^{\mathcal{D}}$ in this case. Anyway, since $\left|S_{m_{1}}\right| \leq 1$ for any $m_{1} \in \mathbb{Z}$, we have

$$
\left|I_{l}^{\mathcal{D}}\right| \leq \frac{\sum_{l_{1}=0}^{l} C_{l}^{l_{1}}}{2^{l}}=1 \text {. }
$$

Hence

$$
\mathcal{I}_{m}^{\mathcal{D}}=\mathrm{e}^{\imath m \alpha} \sum_{l=m}^{N_{T}-m-1} \frac{z^{l}}{2^{l} l !} C_{l}^{(l-m) / 2}\left(\frac{1+(-1)^{l-m}}{2}\right)+\sum_{l=N_{T}-m}^{\infty} \frac{z^{l}}{l !} I_{l}^{\mathcal{D}} .
$$

We also notice that for all $l \in \mathbb{N}$

$$
\left|I_{l}^{\mathcal{D}}\right| \leq 1
$$

Combining (25) and (27), we have

$$
\mathcal{I}_{m}^{\mathcal{C}}-\mathcal{I}_{m}^{\mathcal{D}}=\sum_{l=N_{T}-m}^{\infty} \frac{z^{l}}{l !}\left(I_{l}^{\mathcal{C}}-I_{l}^{\mathcal{D}}\right)
$$

and with (26) and (28)

$$
\left|\mathcal{I}_{m}^{\mathcal{C}}-\mathcal{I}_{m}^{\mathcal{D}}\right| \leq 2 \sum_{l=N_{T}-m}^{\infty} \frac{|z|^{l}}{l !} .
$$

But for $l \geq N_{T}-m$, we have

$$
\frac{|z|^{l}}{l !}=\frac{|z|^{N_{T}-m}|z|^{l-N_{T}+m}}{\left(N_{T}-m\right) ! l(l-1) \cdots\left(N_{T}-m+1\right)} \leq \frac{|z|^{N_{T}-m}}{\left(N_{T}-m\right) !} \frac{|z|^{l-N_{T}+m}}{\left(l-N_{T}+m\right) !} .
$$


Hence setting $l_{1}=l-N_{T}+m$, we get

$$
\left|\mathcal{I}_{m}^{\mathcal{C}}-\mathcal{I}_{m}^{\mathcal{D}}\right| \leq 2 \frac{|z|^{N_{T}-m}}{\left(N_{T}-m\right) !} \sum_{l_{1}=0}^{\infty} \frac{|z|^{l_{1}}}{l_{1} !}
$$

and

$$
\left|\mathcal{I}_{m}^{\mathcal{C}}-\mathcal{I}_{m}^{\mathcal{D}}\right| \leq 2 \mathrm{e}^{|z|} \frac{|z|^{N_{T}-m}}{\left(N_{T}-m\right) !}
$$

By symmetry only positive values of $m$ have to be considered. Hence the error between $G_{N}^{\mathcal{C}}\left(\mathbf{x}, \mathbf{x}^{\prime}\right)$ and $G_{N}^{\mathcal{D}}\left(\mathbf{x}, \mathbf{x}^{\prime}\right)$ is

$$
\left|G_{N}^{\mathcal{C}}\left(\mathbf{x}, \mathbf{x}^{\prime}\right)-G_{N}^{\mathcal{D}}\left(\mathbf{x}, \mathbf{x}^{\prime}\right)\right| \leq \frac{1}{4} 2 \sum_{m=0}^{N}\left|H_{m}^{(1)}\left(k \mathbf{z}_{i}-\mathbf{z}_{j} \mid\right)\right|\left|\mathcal{I}_{m}^{\mathcal{C}}-\mathcal{I}_{m}^{\mathcal{D}}\right|
$$

When $0 \leq m \leq \Lambda$, formula (43) enables us to bound $H_{m}^{(1)}\left(k\left|\mathbf{z}_{i}-\mathbf{z}_{j}\right|\right)$. Otherwise (37) is used, leading to

$$
\begin{aligned}
\left|G_{N}^{\mathcal{C}}\left(\mathbf{x}, \mathbf{x}^{\prime}\right)-G_{N}^{\mathcal{D}}\left(\mathbf{x}, \mathbf{x}^{\prime}\right)\right| \leq & \mathrm{e}^{|z|} \sum_{m=0}^{\Lambda} C_{\tilde{\alpha}} \frac{|z|^{N_{T}-m}}{\left(N_{T}-m\right) !} \\
& +\mathrm{e}^{|z|} \sum_{m=\Lambda+1}^{N} M_{H} \sqrt{\frac{2}{\pi m}}\left(\frac{2 m}{\mathrm{e}|k| \mathbf{z}_{i}-\mathbf{z}_{j} \mid}\right)^{m} \frac{|z|^{N_{T}-m}}{\left(N_{T}-m\right) !} .
\end{aligned}
$$

Moreover, by (38), we have $N_{T}-m \geq \Lambda$,

$$
\left(N_{T}-m\right) ! \geq C_{\min } \sqrt{2 \pi\left(N_{T}-m\right)}\left(\frac{N_{T}-m}{e}\right)^{N_{T}-m}
$$

Hence since $z=\imath k r$,

$$
\begin{aligned}
& \left|G_{N}^{\mathcal{C}}\left(\mathbf{x}, \mathbf{x}^{\prime}\right)-G_{N}^{\mathcal{D}}\left(\mathbf{x}, \mathbf{x}^{\prime}\right)\right| \leq \frac{C_{\tilde{\alpha}} \mathrm{e}^{|z|}}{C_{\min }} \sum_{m=0}^{\Lambda} \frac{1}{\sqrt{2 \pi\left(N_{T}-m\right)}}\left(\frac{\mathrm{e}|k| r}{N_{T}-m}\right)^{N_{T}-m} \\
& \quad+\frac{M_{H} \mathrm{e}^{|z|}}{C_{\min }} \sum_{m=\Lambda+1}^{N} \sqrt{\frac{2}{\pi m}}\left(\frac{2 m}{\mathrm{e}|k| \mathbf{z}_{i}-\mathbf{z}_{j} \mid}\right)^{m} \frac{1}{\sqrt{2 \pi\left(N_{T}-m\right)}}\left(\frac{\mathrm{e}|k| r}{N_{T}-m}\right)^{N_{T}-m}
\end{aligned}
$$

Let us apply Proposition 12 part (ii) to $x=m, y=N$ and $a=\frac{\mathrm{e}|k| \mathbf{z}_{i}-\mathbf{z}_{j} \mid}{2}$. If $m \geq \Lambda+1$, then we verify that $y \geq x \geq a$, and thus

$$
\left(\frac{2 m}{\mathrm{e}|k|\left|\mathbf{z}_{i}-\mathbf{z}_{j}\right|}\right)^{m} \leq\left(\frac{2 N}{\mathrm{e}|k| \mathbf{z}_{i}-\mathbf{z}_{j} \mid}\right)^{N}
$$

We now apply Proposition 12 part (i) to $x=N_{T}-m, y=N$ and $a=\mathrm{e}|k| r$, which satisfy $x \geq y \geq a$, since $n \leq N$ and $N_{T}=2 N+1$. Hence

$$
\left(\frac{\mathrm{e}|k| r}{N_{T}-m}\right)^{N_{T}-m} \leq\left(\frac{\mathrm{e}|k| r}{N}\right)^{N}
$$

so that

$$
\begin{aligned}
\left|G_{N}^{\mathcal{C}}\left(\mathbf{x}, \mathbf{x}^{\prime}\right)-G_{N}^{\mathcal{D}}\left(\mathbf{x}, \mathbf{x}^{\prime}\right)\right| \leq & \frac{C_{\tilde{\alpha}} \mathrm{e}^{|z|}}{C_{\min } \sqrt{2 \pi}}(\Lambda+1)\left(\frac{\mathrm{e}|k| r}{N}\right)^{N} \\
& +\frac{M_{H} \mathrm{e}^{|z|}}{C_{\min } \pi \sqrt{N}}(N-\Lambda)\left(\frac{2 N}{\mathrm{e}|k| \mathbf{z}_{i}-\mathbf{z}_{j} \mid}\right)^{N}\left(\frac{\mathrm{e}|k| r}{N}\right)^{N}
\end{aligned}
$$


Consequently, thanks to (24), the theorem is proved.

The rest of the FMM only consists in linear steps. Consequently, the convergence of the matrix-vector multiplication will also be exponential.

Remark 5. The last theorem proves the convergence of the discretized approximated kernel which is used numerically. Unfortunately, because of roundoff errors, this convergence is not numerically attained. The main argument we give here is that Proposition 14 becomes numerically $S_{0} \approx 1$ and $S_{m}$ is roughly equal to the precision $\epsilon$ of the computer (say $\epsilon=10^{-16}$ ) for $1 \leq|m| \leq N_{T}-1$. Using this in the proof of last theorem, we now get instead of $(27)$

$$
\mathcal{I}_{m}^{\mathcal{D}}=\sum_{l=0}^{m-1} \frac{z^{l}}{l !} \epsilon+\mathrm{e}^{\imath m \alpha} \sum_{l=m}^{N_{T}-m-1} \frac{z^{l}}{2^{l} l !} C_{l}^{(l-m) / 2}\left(\frac{1+(-1)^{l-m}}{2}+\epsilon\right)+\sum_{l=N_{T}-m}^{\infty} \frac{z^{l}}{l !} I_{l}^{\mathcal{D}} .
$$

Hence there exists $C_{m}$ such that

$$
\mathcal{I}_{m}^{\mathcal{D}}=\epsilon C_{m}+e^{\imath m \alpha} \sum_{l=m}^{N_{T}-m-1} \frac{z^{l}}{2^{l} l !} C_{l}^{(l-m) / 2}\left(\frac{1+(-1)^{l-m}}{2}\right)+\sum_{l=N_{T}-m}^{\infty} \frac{z^{l}}{l !} I_{l}^{\mathcal{D}},
$$

and

$$
\mathcal{I}_{m}^{\mathcal{C}}-\mathcal{I}_{m}^{\mathcal{D}}=-\epsilon C_{m}+\sum_{l=N_{T}-m}^{\infty} \frac{z^{l}}{l !}\left(I_{l}^{\mathcal{C}}-I_{l}^{\mathcal{D}}\right)
$$

We recall that

$$
\left|G_{N}^{\mathcal{C}}\left(\mathbf{x}, \mathbf{x}^{\prime}\right)-G_{N}^{\mathcal{D}}\left(\mathbf{x}, \mathbf{x}^{\prime}\right)\right| \leq \frac{1}{2} \sum_{m=0}^{N}\left|H_{m}^{(1)}\left(k\left|\mathbf{z}_{i}-\mathbf{z}_{j}\right|\right)\right|\left|\mathcal{I}_{m}^{\mathcal{C}}-\mathcal{I}_{m}^{\mathcal{D}}\right| .
$$

The convergence of the second term in the right hand side of (30) has been shown in the last theorem. Since $C_{m}$ does not tend to zero when $m$ tends to infinity, and $\epsilon$ is fixed, one concludes that $\sum_{m=0}^{N}\left|H_{m}^{(1)}\left(k\left|\mathbf{z}_{i}-\mathbf{z}_{j}\right|\right)\right| \epsilon\left|C_{m}\right|$ tends to infinity when $N$ tends to infinity. This proves that $\mathcal{I}_{m}^{\mathcal{C}}-\mathcal{I}_{m}^{\mathcal{D}}$ does not numerically converge uniformly. This quantity actually diverges. This is what one can in fact observe numerically when taking $N$ very large.

\section{Moment method matrix of the FMM}

Let us first define a mesh on $\Gamma$. As mentioned in Section $2, \Gamma$ is split into $p$ aggregates: $\Gamma=\bigcup_{i=1}^{p} A_{i}$, with $A_{i} \cap A_{j}=\emptyset$ if $i \neq j$. Each aggregate $A_{i}$ is itself divided into $q$ elements: $A_{i}=\bigcup_{l=1}^{q} \Gamma_{(i, l)}$ with $\Gamma_{(i, l)} \cap \Gamma_{\left(j, l^{\prime}\right)}=\emptyset$ if $i \neq j$ or $l \neq l^{\prime}$. Thus there are $n=p q$ elements $\Gamma_{(i, l)}$ of $\Gamma$. This is the mesh. The two extremities of the element $\Gamma_{(i, l)}$ are denoted by $\mathbf{x}_{(i, l-1)}$ and $\mathbf{x}_{(i, l)}$. Here we use the moment method with the $P_{1}$ finite element basis. Let $\left\{f_{(i, l)}\right\}_{1 \leq i \leq p, 1 \leq l \leq q}$ be the triangular $P_{1}$ finite element basis defined on $\Gamma: f_{(i, l)}$ is affine on each element $\Gamma_{\left(j, l^{\prime}\right)}$, and $f_{(i, l)}\left(\mathbf{x}_{(i, l)}\right)=1, f_{(i, l)}\left(\mathbf{x}_{\left(j, l^{\prime}\right)}\right)=0$ for $j \neq i$ or $l^{\prime} \neq i$. The support of $f_{(i, l)}$ is exactly $\Gamma_{(i, l)} \cup \Gamma_{(i, l+1)}$, with the notation $\Gamma_{(i, q+1)}=\Gamma_{(i+1,1)}$ and $\Gamma_{(p+1,1)}=\Gamma_{(1,1)}$ by periodicity. Let us set $\tilde{A}_{i}:=A_{i} \cup \Gamma_{(i, q+1)}$, so that the support of $f_{(i, l)}$ belongs to $\tilde{A}_{i}$ for all $1 \leq l \leq q$. For $i$ fixed, we also denote by $W_{i}$ the set of all the "remote aggregates", i.e. all $\tilde{A}_{j}$ such that (18) holds for all $\mathbf{x} \in \tilde{A}_{i}, \mathbf{x}^{\prime} \in \tilde{A}_{j} . \widehat{W}_{i}:=\Gamma \backslash W_{i}$ is the complementary set (composed of the "nearby aggregates"). 
Let $Y$ be a function defined on $\Gamma$ that belongs to the $P_{1}$ finite element space described earlier. If $\mathbf{Y}_{(i, l)}:=Y\left(\mathbf{x}_{(i, l)}\right)$, then

$$
Y(\mathbf{x})=\sum_{i=1}^{p} \sum_{l=1}^{q} \mathbf{Y}_{(i, l)} f_{(i, l)}(\mathbf{x})
$$

Then $Y$ is associated with the vector $\mathbf{Y}:=\left\{\mathbf{Y}_{(i, l)}\right\}_{i, l}$ defined by the double index $(i, l)$. In the same way, the elements $\mathbf{A}_{(i, l),\left(j, l^{\prime}\right)}$ of any matrix $\mathbf{A}$ are defined by the two double indexes $(i, l)$ and $\left(j, l^{\prime}\right)$. The matrix-vector multiplication of the matrix $\mathbf{A}$ by the vector $\mathbf{Y}$ is defined by

$$
(\mathbf{A} \cdot \mathbf{Y})_{(i, l)}=\sum_{j=1}^{p} \sum_{l^{\prime}=1}^{q} \mathbf{A}_{(i, j),\left(j, l^{\prime}\right)} \mathbf{Y}_{\left(j, l^{\prime}\right)}
$$

The exact matrix $\mathbf{Z}$ which comes from the moment method discretization of (8) is defined by its components $\mathbf{Z}_{(l, i),\left(l^{\prime}, j\right)}$ :

$$
\begin{array}{r}
\mathbf{Z}_{(l, i),\left(l^{\prime}, j\right)=}=\int_{\Gamma}\left[D\left(f_{(i, l)}\right)+i \zeta K\left(f_{(i, l)}\right)\right. \\
\left.\quad+i K^{t}\left(\zeta f_{(i, l)}\right)+\zeta V\left(\zeta f_{(i, l)}\right)\right](\mathbf{x}) f_{\left(j, l^{\prime}\right)}(\mathbf{x}) d \gamma(\mathbf{x}) .
\end{array}
$$

When $\tilde{A}_{j} \not \subset W_{i}$, the approximate kernel $G_{N}^{\mathcal{D}}$ cannot be used and thus the exact kernel is still employed. The FMM matrix is then

$$
\mathbf{Z}_{(l, i),\left(l^{\prime}, j\right)}^{(N)}=\left\{\begin{array}{l}
\text { formula (31) with the approximate kernel } G_{N}^{\mathcal{D}} \text { if } \tilde{A}_{j} \subset W_{i}, \\
\mathbf{Z}_{(l, i),\left(l^{\prime}, j\right)} \text { otherwise }
\end{array}\right.
$$

In order to prove the convergence of $\mathbf{Z}^{(N)}$, let us begin with the following theorem :

Theorem 6. Let $i$ and $j$ be such that $\tilde{A}_{j} \subset W_{i}$. Then

$$
\begin{gathered}
G_{N}^{\mathcal{D}}\left(\mathbf{x}, \mathbf{x}^{\prime}\right) \stackrel{N \rightarrow \infty}{\longrightarrow} G\left(\mathbf{x}, \mathbf{x}^{\prime}\right), \\
\frac{\partial}{\partial \mathbf{n}(\mathbf{x})} G_{N}^{\mathcal{D}}\left(\mathbf{x}, \mathbf{x}^{\prime}\right) \stackrel{N \rightarrow \infty}{\longrightarrow} \frac{\partial}{\partial \mathbf{n}(\mathbf{x})} G\left(\mathbf{x}, \mathbf{x}^{\prime}\right), \\
\frac{\partial}{\partial \mathbf{n}\left(\mathbf{x}^{\prime}\right)} G_{N}^{\mathcal{D}}\left(\mathbf{x}, \mathbf{x}^{\prime}\right) \stackrel{N \rightarrow \infty}{\longrightarrow} \frac{\partial}{\partial \mathbf{n}\left(\mathbf{x}^{\prime}\right)} G\left(\mathbf{x}, \mathbf{x}^{\prime}\right), \\
\frac{\partial^{2}}{\partial \mathbf{n}(\mathbf{x}) \partial \mathbf{n}\left(\mathbf{x}^{\prime}\right)} G_{N}^{\mathcal{D}}\left(\mathbf{x}, \mathbf{x}^{\prime}\right) \stackrel{N \rightarrow \infty}{\longrightarrow} \frac{\partial^{2}}{\partial \mathbf{n}(\mathbf{x}) \partial \mathbf{n}\left(\mathbf{x}^{\prime}\right)} G\left(\mathbf{x}, \mathbf{x}^{\prime}\right) .
\end{gathered}
$$

All the left hand sides converge absolutely and uniformly with respect to $\mathbf{x} \in \tilde{A}_{i}$ and $\mathbf{x}^{\prime} \in \tilde{A}_{j}$, when $N \rightarrow \infty$.

Proof. From Theorems 2 and $4, G_{N}^{\mathcal{D}}\left(\mathbf{x}, \mathbf{x}^{\prime}\right)$ converges absolutely to $G\left(\mathbf{x}, \mathbf{x}^{\prime}\right)$. The uniform convergence comes from the construction of $W_{i}$ :

$$
\max _{\mathbf{x} \in \tilde{A}_{i}} \frac{\left|\mathbf{x}-\mathbf{z}_{i}\right|}{\left|\mathbf{z}_{i}-\mathbf{z}_{j}\right|}<\frac{1}{1+2 \mathrm{e}}, \max _{\mathbf{x}^{\prime} \in \tilde{A}_{j}} \frac{\left|\mathbf{x}^{\prime}-\mathbf{z}_{j}\right|}{\left|\mathbf{z}_{i}-\mathbf{z}_{j}\right|}<\frac{1}{1+2 \mathrm{e}} .
$$

The expression of $\frac{\partial}{\partial \mathbf{n}(\mathbf{x})} G_{N}^{\mathcal{D}}\left(\mathbf{x}, \mathbf{x}^{\prime}\right), \frac{\partial}{\partial \mathbf{n}\left(\mathbf{x}^{\prime}\right)} G_{N}^{\mathcal{D}}\left(\mathbf{x}, \mathbf{x}^{\prime}\right)$ and $\frac{\partial^{2}}{\partial \mathbf{n}(\mathbf{x}) \partial \mathbf{n}\left(\mathbf{x}^{\prime}\right)} G_{N}^{\mathcal{D}}\left(\mathbf{x}, \mathbf{x}^{\prime}\right)$ is quite similar to that of $G_{N}^{\mathcal{D}}\left(\mathbf{x}, \mathbf{x}^{\prime}\right)$. Consequently, the proof of Lemma 1, Theorem 2 and 
Theorem 4 can be carried over almost unchanged to the case of normal derivatives. The last three convergence results of this theorem follow.

Assume that $\tilde{A}_{j} \subset W_{i}$. From the above theorem, we automatically have

$$
\begin{array}{r}
\left|\int_{\Gamma \times \Gamma}\left[G\left(\mathbf{x}, \mathbf{x}^{\prime}\right)-G_{N}^{\mathcal{D}}\left(\mathbf{x}, \mathbf{x}^{\prime}\right)\right] \zeta(\mathbf{x}) f_{(i, l)}(\mathbf{x}) \zeta\left(\mathbf{x}^{\prime}\right) f_{\left(j, l^{\prime}\right)}\left(\mathbf{x}^{\prime}\right) d \gamma(\mathbf{x}) d \gamma\left(\mathbf{x}^{\prime}\right)\right| \\
\leq \quad\left[\max _{\mathbf{x} \in \tilde{A}_{i}, \mathbf{x}^{\prime} \in \tilde{A}_{j}}\left|G\left(\mathbf{x}, \mathbf{x}^{\prime}\right)-G_{N}^{\mathcal{D}}\left(\mathbf{x}, \mathbf{x}^{\prime}\right)\right|\right] \\
\cdot \int_{\tilde{A}_{i} \times \tilde{A}_{j}} \zeta(\mathbf{x}) f_{(i, l)}(\mathbf{x}) \zeta\left(\mathbf{x}^{\prime}\right) f_{\left(j, l^{\prime}\right)}\left(\mathbf{x}^{\prime}\right) d \gamma(\mathbf{x}) d \gamma\left(\mathbf{x}^{\prime}\right) .
\end{array}
$$

since the support of $f_{(i, l)}$ belongs to $\tilde{A}_{i}$ and that of $f_{\left(j, l^{\prime}\right)}$ belongs to $\tilde{A}_{j}$. Thanks to the uniform convergence in Theorem 6 , we have

$$
\max _{\mathbf{x} \in \tilde{A}_{i}, \mathbf{x}^{\prime} \in \tilde{A}_{j}}\left|G\left(\mathbf{x}, \mathbf{x}^{\prime}\right)-G_{N}^{\mathcal{D}}\left(\mathbf{x}, \mathbf{x}^{\prime}\right)\right| \stackrel{N \rightarrow \infty}{\longrightarrow} 0
$$

Thus

$$
\begin{gathered}
\int_{\Gamma \times \Gamma} G_{N}^{\mathcal{D}}\left(\mathbf{x}, \mathbf{x}^{\prime}\right) \zeta(\mathbf{x}) f_{(i, l)}(\mathbf{x}) \zeta\left(\mathbf{x}^{\prime}\right) f_{\left(j, l^{\prime}\right)}\left(\mathbf{x}^{\prime}\right) d \gamma(\mathbf{x}) d \gamma\left(\mathbf{x}^{\prime}\right) \\
\stackrel{N \rightarrow \infty}{\longrightarrow} \int_{\Gamma} V\left(\zeta f_{(i, l)}\right)\left(\mathbf{x}^{\prime}\right) \zeta\left(\mathbf{x}^{\prime}\right) f_{\left(j, l^{\prime}\right)}\left(\mathbf{x}^{\prime}\right) d \gamma\left(\mathbf{x}^{\prime}\right)
\end{gathered}
$$

We have the same convergence for the derivatives $\frac{\partial}{\partial \mathbf{n}(\mathbf{x})}, \frac{\partial}{\partial \mathbf{n}\left(\mathbf{x}^{\prime}\right)}$ and $\frac{\partial^{2}}{\partial \mathbf{n}(\mathbf{x}) \partial \mathbf{n}\left(\mathbf{x}^{\prime}\right)}$. In this latter case the integral for $N=\infty$ must be taken in the finite part sense $([7])$. From these convergence results, we can infer that

$$
\mathbf{Z}_{(l, i),\left(l^{\prime} . j\right)}^{(N)} \stackrel{N \rightarrow \infty}{\longrightarrow} \mathbf{Z}_{(l, i),\left(l^{\prime}, j\right)}
$$

Now if $\tilde{A}_{j} \subset \widehat{W}_{i}$, then $\mathbf{Z}_{(l, i),\left(l^{\prime} . j\right)}^{(N)}=\mathbf{Z}_{(l, i),\left(l^{\prime}, j\right)}$ from formula (32). Therefore we have proved the next theorem.

Theorem 7. We have $\mathbf{Z}^{(N)} \stackrel{N \rightarrow \infty}{\longrightarrow} \mathbf{Z}$ in any norm.

We conclude this section by giving the explicit form of $\mathbf{Z}_{(l, i),\left(l^{\prime} . j\right)}^{(N)}$ when $j$ is such that $\tilde{A}_{j} \subset W_{i}$. By setting

$$
\begin{gathered}
\mathcal{V}_{(i, l)}^{ \pm}(\theta)=\int_{\Gamma} \exp \left( \pm \imath k\left(\mathbf{z}_{i}-\mathbf{x}\right) \cdot \mathbf{U}(\theta)\right) f_{(i, l)}(\mathbf{x}) \zeta(\mathbf{x}) d \gamma(\mathbf{x}), \\
\mathcal{W}_{(i, l)}^{ \pm}(\theta)=\int_{\Gamma} \pm \imath k \mathbf{n}(\mathbf{x}) \cdot \mathbf{U}(\theta) \exp \left( \pm \imath k\left(\mathbf{z}_{i}-\mathbf{x}\right) \cdot \mathbf{U}(\theta)\right) f_{(i, l)}(\mathbf{x}) d \gamma(\mathbf{x}),
\end{gathered}
$$

we obtain with $\theta_{n_{T}}=\frac{2 \pi n_{T}}{N_{T}}$,

$$
\begin{aligned}
\mathbf{Z}_{(l, i),\left(l^{\prime}, j\right)}^{(N)}= & -\frac{1}{2 \pi N_{T}} \sum_{n_{T}=1}^{N_{T}}\left\{\mathcal{W}_{\left(j, l^{\prime}\right)}^{+}\left(\theta_{n_{T}}\right) \mathcal{W}_{(i, l)}^{-}\left(\theta_{n_{T}}\right)\right. \\
& +i\left(\mathcal{W}_{\left(j, l^{\prime}\right)}^{+}\left(\theta_{n_{T}}\right) \mathcal{V}_{(i, l)}^{-}\left(\theta_{n_{T}}\right)+\mathcal{V}_{\left(j, l^{\prime}\right)}^{+}\left(\theta_{n_{T}}\right) \mathcal{W}_{(i, l)}^{-}\left(\theta_{n_{T}}\right)\right) \\
& \left.-\mathcal{V}_{\left(j, l^{\prime}\right)}^{+}\left(\theta_{n_{T}}\right) \mathcal{V}_{(i, l)}^{-}\left(\theta_{n_{T}}\right)\right\} \tau_{i j}^{(N)}\left(\theta_{n_{T}}\right) .
\end{aligned}
$$




\section{Matrix-VECtor MUltiplication}

The multiplication of $\mathbf{Z}$ by a vector $\mathbf{Y}=\left(\mathbf{Y}_{(l, i)}\right)_{1 \leq i \leq p, 1 \leq l \leq q}$ is done as follows:

$$
(\mathbf{Z} \cdot \mathbf{Y})_{(l, i)} \approx\left(\tilde{\mathbf{Z}}^{(N)} \cdot \mathbf{Y}\right)_{(l, i)}=\underbrace{\sum_{\tilde{A}_{j} \in \widehat{W}_{i}} \sum_{l^{\prime}=1}^{q} \mathbf{Z}_{(l, i),\left(l^{\prime}, j\right)} \mathbf{Y}_{\left(l^{\prime}, j\right)}}_{\text {"nearby" aggregates }}+\underbrace{\left.\sum_{\tilde{A}_{j} \in W_{i}} \sum_{l^{\prime}=1}^{q} \tilde{\mathbf{Z}}_{(l, i),\left(l^{\prime}, j\right)}^{(N)} \mathbf{Y}_{\left(l^{\prime}, j\right)}\right)}_{\text {"remote" aggregates }} .
$$

The first part of the right hand side of above equation can be viewed as the multiplication of a sparse matrix $\mathcal{S}$ by $\mathbf{Y}$. The matrix $\mathcal{S}$ is sparse since the number of nearby aggregates is equal to about 7 , provided the shape of $\Omega_{i}$ is convex. If $\Omega_{i}$ is not convex, there might be a few more nearby aggregates. The components of $\mathcal{S}$ are computed in the standard way since Rokhlin's formula (6) does not hold for nearby aggregates. To examine the second part of the formula, let us first set

$$
\begin{aligned}
S_{\mathcal{V}}^{j}\left(\theta_{n_{T}}\right) & =\sum_{l^{\prime}=1}^{q} \mathcal{V}_{\left(j, l^{\prime}\right)}^{+}\left(\theta_{n_{T}}\right) \mathbf{Y}_{\left(l^{\prime}, j\right)}, \\
S_{\mathcal{W}}^{j}\left(\theta_{n_{T}}\right) & =\sum_{l^{\prime}=1}^{q} \mathcal{W}_{\left(j, l^{\prime}\right)}^{+}\left(\theta_{n_{T}}\right) \mathbf{Y}_{\left(l^{\prime}, j\right)}, \\
T_{\mathcal{V}}^{i}\left(\theta_{n_{T}}\right) & =\sum_{\tilde{A}_{j} \in W_{i}} \tau_{i j}^{(N)}\left(\theta_{n_{T}}\right) S_{\mathcal{V}}^{j}\left(\theta_{n_{T}}\right), \\
T_{\mathcal{W}}^{i}\left(\theta_{n_{T}}\right) & =\sum_{\tilde{A}_{j} \in W_{i}} \tau_{i j}^{(N)}\left(\theta_{n_{T}}\right) S_{\mathcal{W}}^{j}\left(\theta_{n_{T}}\right) .
\end{aligned}
$$

Then a straightforward calculation shows that

$$
\begin{aligned}
(\mathbf{Z} \cdot \mathbf{Y})_{(l, i)} \approx(\mathcal{S} \cdot \mathbf{Y})_{(l, i)}- & \frac{1}{2 \pi N_{T}} \sum_{n_{T}=1}^{N_{T}}\left\{\mathcal{W}_{(i, l)}^{-}\left(\theta_{n_{T}}\right) T_{\mathcal{W}}^{i}\left(\theta_{n_{T}}\right)\right. \\
& +i\left(\mathcal{V}_{(i, l)}^{-}\left(\theta_{n_{T}}\right) T_{\mathcal{W}}^{i}\left(\theta_{n_{T}}\right)+\mathcal{W}_{(i, l)}^{-}\left(\theta_{n_{T}}\right) T_{\mathcal{V}}^{i}\left(\theta_{n_{T}}\right)\right) \\
& \left.-\mathcal{V}_{(i, l)}^{-}\left(\theta_{n_{T}}\right) T_{\mathcal{V}}^{i}\left(\theta_{n_{T}}\right)\right\} .
\end{aligned}
$$

The main difficulty we face in studying Rokhlin's method lies in the fact that, even if from a theoretical point of view (see Theorems 2, 4, 6 and 7) the greater $N$ the more accurate the approximation, $N$ must (in numerical simulations) belong to a fixed range of integers. If $N$ is too small, the overall accuracy is not good, which is quite logical. But if $N$ is too large, then (6) is not numerically accurate. We refer here to Remark 5. Hopefully, there is a range of integer values $N$ such that the accuracy of Rokhlin's formula (6) is quite good (double precision is reached).

Theorems 2 and 4 are not used numerically to compute the best $N$ since these theorems assume that $N>C|k|^{2} \max _{i, j}\left|\mathbf{z}_{i}-\mathbf{z}_{j}\right|^{2}$. Numerically the integer $N$ such that (6) provides the best accuracy is always bracketed between the two following values:

$$
|k| \max _{i} \min _{\mathbf{x}^{\prime} \in \tilde{A}_{j} \subset W_{i}}\left|\mathbf{x}^{\prime}-\mathbf{z}_{i}\right|, \quad|k| \max _{i, j}\left|\mathbf{z}_{i}-\mathbf{z}_{j}\right| .
$$

To end this section, let us explain briefly why (34) leads to an $O\left(n^{3 / 2}\right)$ matrixvector multiplication, as stated in the Introduction. To do so, we shall give the link between all the quantities that have been introduced before. If $L$ is the length of the 
obstacle $\Omega_{i}$, then the number $n$ of mesh intervals used to discretize $\Gamma$ satisfies $n=$ $O(|k| L)$. Here we have assumed that the number of mesh interval per wavelength is fixed (usually between 6 and 10). We recall that $p$ is the number of aggregates and $q$ is the number of mesh intervals in each aggregate, with $n=p q$. If one chooses $p, q$ so that $p \approx q$, then $p \approx q=O(\sqrt{n})$. If $n$ is large (say 1,000 ), the optimal number $N$ is approximately $N \approx|k| \max _{i} \min _{\mathbf{x}^{\prime} \in \tilde{A}_{j} \subset W_{i}}\left|\mathbf{x}^{\prime}-\mathbf{z}_{i}\right|=O(q)=O(\sqrt{n})$. And finally $N_{T}=2 N+1=O(\sqrt{n})$. The calculation can be split into parts

- The first calculation does not depend on the vector $\mathbf{Y}$, and thus need be performed only once. This consists in computing $\mathcal{V}_{(i, l)}^{ \pm}\left(\theta_{n_{T}}\right)$ and $\mathcal{W}_{(i, l)}^{ \pm}\left(\theta_{n_{T}}\right)$ (for $1 \leq i \leq p, 1 \leq l \leq q$ and $\left.1 \leq n_{T} \leq N_{T}\right)$, which requires $p q N_{T}=O\left(n^{3 / 2}\right)$ operations. Next $\tau_{i j}^{(N)}\left(\theta_{n_{T}}\right)$ (for $1 \leq i, j \leq p$ and $1 \leq n_{T} \leq N_{T}$ ) leads to $p^{2} N_{T} N=O\left(n^{2}\right)$ operations. In fact, this number can be reduced by using the Fast Fourier Transform, leading to $O\left(n^{3 / 2} \log n\right)$ operations.

- The second calculation is the matrix-vector multiplication step itself. First, one has to compute $S_{\mathcal{V}}^{j}\left(\theta_{n_{T}}\right), S_{\mathcal{W}}^{j}\left(\theta_{n_{T}}\right)$ (for $1 \leq j \leq p$ and $1 \leq n_{T} \leq N_{T}$ ) and $T_{\mathcal{V}}^{i}\left(\theta_{n_{T}}\right), T_{\mathcal{W}}^{i}\left(\theta_{n_{T}}\right)$ (for $1 \leq i \leq p$ and $\left.1 \leq n_{T} \leq N_{T}\right)$, which both require $O\left(n^{3 / 2}\right)$ operations. Thanks to the remark at the end of Section 4 , the average number of nonzero elements in each row of the matrix $\mathcal{S}$ is about $7 q=O(\sqrt{n})$. Thus $\mathcal{S}$ has only $O\left(n^{3 / 2}\right)$ nonzero elements, which proves that $\mathcal{S}$ is sparse. We conclude that the matrix-vector multiplication $\mathcal{S} \cdot \mathbf{Y}$ requires $O\left(n^{3 / 2}\right)$ operations. The remaining term of formula (34) clearly leads to $O\left(n^{3 / 2}\right)$ operations.

Let us conclude this section by giving the cost of the FMM in terms of the size of the obstacle as measured in wavelengths. We noticed earlier that $n=$ $O(|k| L)$. Hence $n=O\left(\frac{L}{\lambda}\right)$, where $\lambda$ is the wavelength, so that the matrix-vector multiplication requires $O\left(\left(\frac{L}{\lambda}\right)^{3 / 2}\right)$ operations.

\section{Conclusion}

In this paper, we have shown the convergence of the FMM in two dimensions (Theorems 2, 4 and 6). We assert that the same kind of theorem can be proved in $\mathbb{R}^{3}$. In order to explain how to implement the FMM, we considered a specific problem. We took it as general as possible (namely the impedance boundary condition) with the moment method for the discretization. The FMM can obviously be applied to other boundary conditions and to other numerical methods (collocation method, ...). Here the convergence of the FMM with moment method has been proved (Theorem 7).

The domain of validity of the approximation (6) for $\mathbf{x} \in A_{i}$ and $\mathbf{x}^{\prime} \in A_{j}$, as stated in Theorems 2 and 4 , is roughly $|i-j| \geq 4$. This condition does not appear to be optimal since in numerical simulations we have noticed the convergence of (6) as soon as $|i-j| \geq 2$ (for smooth obstacles).

As far as numerical issues are concerned, some preliminary computations can be done first. They consist in constructing the sparse matrix $\mathcal{S}$ and then computing some numbers $\left(\mathcal{V}_{(i, l)}^{ \pm}\left(\theta_{n_{T}}\right), \mathcal{W}_{(i, l)}^{ \pm}\left(\theta_{n_{T}}\right)\right.$ and $\left.\tau_{i j}^{(N)}\left(\theta_{n_{T}}\right)\right)$. The CPU cost is much lower than the construction of the dense matrix $\mathbf{Z}$, as done for the classical inversion of Z. Next the number of operations required for each matrix-vector multiplication is $O\left(n^{3 / 2}\right)$ or $O\left(\left(\frac{L}{\lambda}\right)^{3 / 2}\right)$, which is much lower than $O\left(n^{2}\right)$ or $O\left(\left(\frac{L}{\lambda}\right)^{2}\right)$. 
In this paper, we also showed that the choice of $N$ is crucial in the FMM. First, the accuracy of (4) depends on $N$. Secondly, the CPU cost is linearly related to $N$. Thus before starting the FMM in a Helmholtz solver, one should compute the best value for $N$.

\section{Appendix A: Useful formulae on Hankel functions}

We only recall here the formulae for Bessel and Hankel functions that are necessary to understand the FMM.

- To begin, let us give two important relations [1, formulae 9.1.5 and 9.1.6]

$$
H_{-m}^{(1)}(z)=(-1)^{m} H_{m}^{(1)}(z), J_{-m}(z)=(-1)^{m} J_{m}(z) .
$$

- For the convergence analysis we need some uniform bounds on the Bessel and Hankel functions.

Proposition 8. Let $R$ and $R^{\prime}$ be two positive constants with $R>R^{\prime}$. Then there exists a constant $C$ depending only on $R$ and $R^{\prime}$ such that

$$
\frac{m_{J}}{\sqrt{2 \pi|n|}}\left(\frac{\mathrm{e}|z|}{2|n|}\right)^{|n|} \leq\left|J_{n}(z)\right| \leq \frac{M_{J}}{\sqrt{2 \pi|n|}}\left(\frac{\mathrm{e}|z|}{2|n|}\right)^{|n|}
$$

for all $|z| \leq R,|n|>C|z|^{2}$, and

$$
m_{H} \sqrt{\frac{2}{\pi|n|}}\left(\frac{2|n|}{\mathrm{e}|z|}\right)^{|n|} \leq\left|H_{n}^{(1)}(z)\right| \leq M_{H} \sqrt{\frac{2}{\pi|n|}}\left(\frac{2|n|}{\mathrm{e}|z|}\right)^{|n|}
$$

for all $R^{\prime} \leq|z| \leq R$ and $|n|>C|z|^{2}$.

The choices of $R$ and $R^{\prime}$ are given in the beginning of Section 4 .

Proof. Thanks to (35), we only have to consider the case when $n$ is positive.

- From [1, formula 9.1.10], the Bessel function has the following expansion

$$
J_{n}(z)=\frac{1}{n !}\left(\frac{z}{2}\right)^{n} \sum_{m=0}^{\infty} \frac{(-1)^{m} n !}{m !(n+m) !}\left(\frac{z}{2}\right)^{2 m} .
$$

Hence

$$
\left|\frac{J_{n}(z)}{\frac{1}{n !}\left(\frac{z}{2}\right)^{n}}-1\right| \leq \sum_{m=1}^{\infty} \frac{n !}{m !(n+m) !}\left(\frac{|z|}{2}\right)^{2 m} .
$$

The Stirling formula enables us to write

$$
n ! \stackrel{n \rightarrow \infty}{\sim} \sqrt{2 \pi n}\left(\frac{n}{\mathrm{e}}\right)^{n} .
$$

Thus, there exist two constants $C_{\min }$ and $C_{\max }$ such that for all $n \in \mathbb{N}^{*}$

$$
C_{\min } \sqrt{2 \pi n}\left(\frac{n}{\mathrm{e}}\right)^{n} \leq n ! \leq C_{\max } \sqrt{2 \pi n}\left(\frac{n}{\mathrm{e}}\right)^{n} .
$$

Since $m \geq 0$, we conclude that

$\frac{n !}{(n+m) !} \leq \frac{C_{\max }}{C_{\min }} \sqrt{\frac{n}{n+m}} \frac{n^{n} \mathrm{e}^{m}}{(n+m)^{n+m}} \leq \frac{C_{\max }}{C_{\min }} \frac{n^{n} \mathrm{e}^{m}}{(n+m)^{n+m}}$.

From Proposition 11 part (ii), we obtain

$$
\frac{n !}{(n+m) !} \leq \frac{C_{\max }}{C_{\min }} \frac{1}{n^{m}},
$$


and

$$
\sum_{m=1}^{\infty} \frac{n !}{m !(n+m) !}\left(\frac{|z|}{2}\right)^{2 m} \leq \frac{C_{\max }}{C_{\min }} \sum_{m=1}^{\infty} \frac{1}{m !}\left(\frac{|z|^{2}}{4 n}\right)^{m} .
$$

If $n \geq \frac{|z|^{2}}{2}$, then $\frac{|z|^{2}}{4 n} \leq \frac{1}{2}$, which implies that

$$
\left|\frac{J_{n}(z)}{\frac{1}{n !}\left(\frac{z}{2}\right)^{n}}-1\right| \leq \frac{C_{\max }}{C_{\min }} \frac{|z|^{2}}{4 n} \sum_{m=0}^{\infty} \frac{1}{2^{m}} \leq \frac{C_{\max }}{C_{\min }} \frac{|z|^{2}}{2 n} .
$$

Let $C=\max \left(\frac{1}{2}, \frac{C_{\max }}{C_{\min }}\right)$ so that for $n>C|z|^{2}$ we have $\frac{C_{\max }}{C_{\min }} \frac{|z|^{2}}{2 n}<\frac{1}{2}$.

Hence, using (38), we have shown (36) with $m_{J}=\frac{1}{2 C_{\max }}$ and $M_{J}=\frac{3}{2 C_{\min }}$.

- From [1, formula 9.1.11], on can write

$$
H_{n}^{(1)}(z)=\left(1+\frac{2 \imath}{\pi}\left(\gamma+\log \left(\frac{z}{2}\right)\right)\right) J_{n}(z)+\frac{\imath}{\pi} T_{n}^{(1)}(z)+\frac{\imath}{\pi} T_{n}^{(2)}(z),
$$

where $\gamma$ is the Euler constant $(\gamma=0.5772157 \ldots)$, and

$$
\begin{gathered}
T_{n}^{(1)}(z)=-(n-1) !\left(\frac{2}{z}\right)^{n} \sum_{m=0}^{n-1} \frac{(n-m-1) !}{m !(n-1) !}\left(\frac{z}{2}\right)^{2 m}, \\
T_{n}^{(2)}(z)=-\sum_{m=0}^{+\infty} \frac{(-1)^{m}\left(\frac{z}{2}\right)^{n+2 m}}{m !(m+n) !}(\psi(m)+\psi(m+n)),
\end{gathered}
$$

with $\psi(m):=\frac{1}{1}+\frac{1}{2}+\ldots+\frac{1}{m}$ (for $m \in \mathbb{N}^{*}$ ), and $\psi(0)=0$. Thus

$$
\begin{aligned}
\left|\frac{H_{n}^{(1)}(z)}{-\frac{\imath}{\pi}(n-1) !\left(\frac{2}{z}\right)^{n}}-1\right| \leq & \left|1+\frac{2 \imath}{\pi}\left(\gamma+\log \left(\frac{z}{2}\right)\right)\right|\left|\frac{\pi J_{n}(z)}{(n-1) !\left(\frac{2}{z}\right)^{n}}\right| \\
& +\left|\frac{T_{n}^{(1)}(z)}{-(n-1) !\left(\frac{2}{z}\right)^{n}}-1\right|+\left|\frac{T_{n}^{(2)}(z)}{(n-1) !\left(\frac{2}{z}\right)^{n}}\right| .
\end{aligned}
$$

Let us assume that $n>C|z|^{2}$. Then, (36) provides an upper bound of the Bessel function

$$
\left|J_{n}(z)\right| \leq \frac{M_{J}}{\sqrt{2 \pi n}}\left(\frac{\mathrm{e}|z|}{2 n}\right)^{n} .
$$

Henceforth, from (38)

$\left|\frac{\pi J_{n}(z)}{(n-1) !\left(\frac{2}{z}\right)^{n}}\right| \leq \frac{\pi M_{J}}{\sqrt{2 \pi n}} \frac{n}{n !}\left(\frac{|z|}{2}\right)^{2 n}\left(\frac{\mathrm{e}}{n}\right)^{n} \leq \frac{M_{J}}{2 C_{\min }}\left(\frac{\mathrm{e}|z|}{2 n}\right)^{2 n}$.

Next, we have

$$
\left|\frac{T_{n}^{(1)}(z)}{-(n-1) !\left(\frac{2}{z}\right)^{n}}-1\right| \leq \sum_{m=1}^{n-1} \frac{(n-m-1) !}{m !(n-1) !}\left(\frac{|z|}{2}\right)^{2 m} .
$$

In order to bound this term, we first notice that

$$
\frac{(n-1-n) !}{(n-1) !} \leq \frac{C_{\max }}{C_{\min }} \frac{(n-1-m)^{n-1-m} \mathrm{e}^{m}}{(n-1)^{n-1}} .
$$


Doing as in the proof of Proposition 11, one can show that

$$
\frac{(n-1-m)^{n-1-m}}{(n-1)^{n-1}} \leq \frac{1}{(n-1)^{m}}
$$

Consequently

$$
\left|\frac{T_{n}^{(1)}(z)}{-(n-1) !\left(\frac{2}{z}\right)^{n}}-1\right| \leq \frac{C_{\max }}{C_{\min }} \sum_{m=1}^{\infty} \frac{1}{m !}\left(\frac{\mathrm{e}^{2}|z|^{2}}{4(n-1)}\right)^{m} .
$$

Since $R^{\prime} \leq|z| \leq R$, there exists a constant again denoted by $C$ such that for $n>C|z|^{2}$, we have $\frac{\mathrm{e}^{2}|z|^{2}}{4(n-1)}<\frac{1}{2}$. Thus

$$
\left|\frac{T_{n}^{(1)}(z)}{-(n-1) !\left(\frac{2}{z}\right)^{n}}-1\right| \leq \frac{C_{\max }}{C_{\min }} \frac{\mathrm{e}^{2}|z|^{2}}{4(n-1)} \sum_{m=0}^{\infty} \frac{1}{2^{m}} \leq \frac{C_{\max }}{C_{\min }} \frac{\mathrm{e}^{2}|z|^{2}}{2(n-1)} .
$$

Since $\psi(p) \leq p$ for $p \in \mathbb{N}$, one may write

$$
\left|\frac{T_{n}^{(2)}(z)}{(n-1) !\left(\frac{2}{z}\right)^{n}}\right| \leq \frac{1}{(n-1) !}\left(\frac{|z|}{2}\right)^{2 n} \sum_{m=0}^{+\infty} \frac{(2 m+n)}{m !(m+n) !}\left(\frac{|z|}{2}\right)^{2 m}
$$

Clearly we have $2 m+n \leq 2(m+n)$, and

$$
\begin{aligned}
\left|\frac{T_{n}^{(2)}(z)}{(n-1) !\left(\frac{2}{z}\right)^{n}}\right| & \leq \frac{2}{(n-1) !}\left(\frac{|z|}{2}\right)^{2 n} \sum_{m=0}^{+\infty} \frac{1}{m !(m+n-1) !}\left(\frac{|z|}{2}\right)^{2 m} \\
& \leq \frac{2}{((n-1) !)^{2}}\left(\frac{|z|}{2}\right)^{2 n} \sum_{m=0}^{+\infty} \frac{1}{m !}\left(\frac{|z|}{2}\right)^{2 m} \\
& =\frac{2}{((n-1) !)^{2}}\left(\frac{|z|}{2}\right)^{2 n} \mathrm{e}^{\frac{|z|^{2}}{4}} .
\end{aligned}
$$

From $(38)$, we have $(n-1) !=\frac{n !}{n} \geq C_{\min } \sqrt{\frac{2 \pi}{n}}\left(\frac{n}{\mathrm{e}}\right)^{n}$. Thus

$$
\left|\frac{T_{n}^{(2)}(z)}{(n-1) !\left(\frac{2}{z}\right)^{n}}\right| \leq \frac{2 n}{\pi C_{\min }}\left(\frac{\mathrm{e}|z|}{2 n}\right)^{2 n} \mathrm{e}^{\frac{|z|^{2}}{4}} .
$$

Combining (39), (40) and (41), we see that there exists a constant again denoted by $C$ such that for all $n>C|z|^{2}$ we have

$$
\left|\frac{H_{n}^{(1)}(z)}{-\frac{\imath}{\pi}(n-1) !\left(\frac{2}{z}\right)^{n}}-1\right| \leq \frac{1}{2} .
$$

Consequently, (37) holds with $m_{H}=\frac{1}{2 C_{\max }}$ and $M_{H}=\frac{3}{2 C_{\min }}$.

- Let us now consider the Bessel function when $|z| \leq R$ and $|n| \leq C|z|^{2}$. $J_{n}(z)$ is well defined everywhere in this set. In addition, the mapping $(n, z) \mapsto J_{n}(z)$ is continuous. Hence, since the set of all $(n, z)$ such that $|z| \leq R$ and $|n| \leq C|z|^{2}$ is compact, we conclude that there exists $C_{\tilde{\beta}}$ depending only on $R$ and $C$ such that

$$
\left|J_{n}(z)\right| \leq C_{\tilde{\beta}} \quad \forall|z| \leq R, \forall|n| \leq C|z|^{2} .
$$




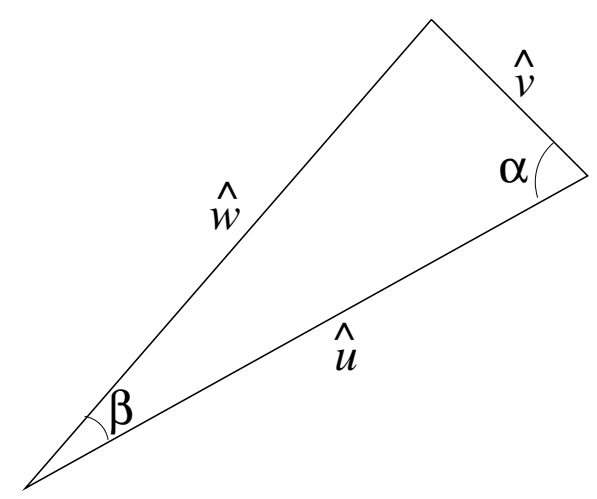

Figure 4. Link between $\widehat{u}, \widehat{v}, \widehat{w}$ and $\alpha, \beta$.

In the same way, one can show that there exists a constant $C_{\tilde{\alpha}}$ such that

$$
\left|H_{n}^{(1)}(z)\right| \leq C_{\tilde{\alpha}} \quad \forall R^{\prime} \leq|z| \leq R, \forall|n| \leq C|z|^{2} .
$$

- The Bessel function $J_{m}$ can be put into an integral form. From formula (9.1.21) in [1], one can easily show that

$$
J_{m}(z)=\frac{1}{2 \pi} \int_{0}^{2 \pi} \exp (\imath z \cos \theta) \exp (\imath m \theta) \exp (-\imath m \pi / 2) d \theta .
$$

- The two formulae (9.1.42) and (9.1.43) in ([1]) yield

$$
\exp (\imath z \sin \theta)=\sum_{m \in \mathbb{Z}} J_{m}(z) \exp (\imath m \theta) .
$$

- The Graf formula is at the root of all the multipole methods. It reads for $u, v, w \in \mathbb{C}[1$, formula 9.1.79]

$$
H_{p_{1}}^{(1)}(w) \exp \left(\imath p_{1} \beta\right)=\sum_{q_{1}=-\infty}^{\infty} H_{p_{1}+q_{1}}^{(1)}(u) J_{q_{1}}(v) \exp \left(\imath q_{1} \alpha\right) \text { if }|v|<|u| .
$$

If $u=k \widehat{u}, v=k \widehat{v}, w=k \widehat{w}$ where $\widehat{u}, \widehat{v}, \widehat{w}$ are positive numbers and $k \in \mathbb{C}$, then $\alpha$ and $\beta$ are positive numbers. Moreover, they represent some angles, as depicted in Figure 4. By (36) and (37), the condition $|v|<|u|$ in (46) implies the convergence of the expansion in the right hand side of (46).

\section{Appendix B: Some multipole formulae}

Let $D_{\mathbf{z}_{i}}, D_{\mathbf{x}^{\prime}}, D_{\mathbf{z}_{j}}$ be three balls with centers respectively $\mathbf{z}_{i}, \mathbf{x}^{\prime}$ and $\mathbf{z}_{j}$ (see Figure 1) chosen so that $D_{\mathbf{x}^{\prime}} \subset D_{\mathbf{z}_{j}}$ and $D_{\mathbf{z}_{i}} \cap D_{\mathbf{z}_{j}}=\emptyset$.

Proposition 9. $\quad$ (i) Let $\Phi$ be a function defined outside $D_{\mathbf{x}^{\prime}}$. We assume that $\Phi$ can be expanded as

$$
\Phi(\mathbf{x})=\sum_{m \in \mathbb{Z}} \beta_{m} H_{m}^{(1)}\left(k\left|\mathbf{x}-\mathbf{x}^{\prime}\right|\right) \exp \left(\imath m \arg \left(\mathbf{x}-\mathbf{x}^{\prime}\right)\right) .
$$

Moreover assume that the expansion

$$
\sum_{l \in \mathbb{Z}} \exp \left(-\imath l \arg \left(\mathbf{x}^{\prime}-\mathbf{z}_{j}\right)\right) \beta_{m-l} J_{l}\left(k\left|\mathbf{x}^{\prime}-\mathbf{z}_{j}\right|\right):=\tilde{\beta}_{m}
$$


converges for all $m \in \mathbb{Z}$, and that $\sum_{m \in \mathbb{Z}}\left|\tilde{\beta}_{m} H_{m}^{(1)}\left(k\left|\mathbf{x}-\mathbf{z}_{j}\right|\right)\right|$ converges for all $\mathbf{x}$ outside $D_{\mathbf{z}_{j}}$. Then outside $D_{\mathbf{z}_{j}}$, $\Phi$ can be written as

$$
\Phi(\mathbf{x})=\sum_{m \in \mathbb{Z}} \tilde{\beta}_{m} H_{m}^{(1)}\left(k\left|\mathbf{x}-\mathbf{z}_{j}\right|\right) \exp \left(\imath m \arg \left(\mathbf{x}-\mathbf{z}_{j}\right)\right) .
$$

(ii) Let $\Phi$ be a function defined outside $D_{\mathbf{z}_{j}}$. We assume that $\Phi$ can be expanded as

$$
\Phi(\mathbf{x})=\sum_{m \in \mathbb{Z}} \alpha_{m} H_{m}^{(1)}\left(k\left|\mathbf{x}-\mathbf{z}_{j}\right|\right) \exp \left(\imath m \arg \left(\mathbf{x}-\mathbf{z}_{j}\right)\right)
$$

Moreover assume that the expansion

$$
\sum_{l \in \mathbb{Z}} \exp \left(-\imath l\left(\arg \left(\mathbf{z}_{i}-\mathbf{z}_{j}\right)-\pi\right)\right) \alpha_{m-l} H_{l}^{(1)}\left(k\left|\mathbf{z}_{i}-\mathbf{z}_{j}\right|\right):=\tilde{\alpha}_{m}
$$

converges for all $m \in \mathbb{Z}$, and that $\sum_{m \in \mathbb{Z}}\left|\tilde{\beta}_{m} J_{m}\left(k\left|\mathbf{x}-\mathbf{z}_{i}\right|\right)\right|$ converges for all $\mathbf{x} \in D_{\mathbf{z}_{i}}$. Then inside $D_{\mathbf{z}_{i}}$, $\Phi$ can be written as

$$
\Phi(\mathbf{x})=\sum_{m \in \mathbb{Z}} \tilde{\alpha}_{m} J_{m}\left(k\left|\mathbf{x}-\mathbf{z}_{i}\right|\right) \exp \left(\imath m \arg \left(\mathbf{x}-\mathbf{z}_{i}\right)\right)
$$

Proof. The two items are proved in a similar way based on the Graf formula (46). We only give details of the proof of the second item. Thanks to the assumptions on the sequences $\alpha_{m}$ and $\tilde{\alpha}_{m}$, the following function

$$
\begin{aligned}
& \Psi(\mathbf{x}):= \sum_{m \in \mathbb{Z}} \tilde{\alpha}_{m} J_{m}\left(k\left|\mathbf{x}-\mathbf{z}_{i}\right|\right) \exp \left(\imath m \arg \left(\mathbf{x}-\mathbf{z}_{i}\right)\right) \\
&= \sum_{m, l \in \mathbb{Z}} \exp \left(-\imath l\left(\arg \left(\mathbf{z}_{i}-\mathbf{z}_{j}\right)-\pi\right)\right) \alpha_{m-l} \\
& \cdot H_{l}^{(1)}\left(k\left|\mathbf{z}_{i}-\mathbf{z}_{j}\right|\right) J_{m}\left(k\left|\mathbf{x}-\mathbf{z}_{i}\right|\right) \exp \left(\imath m \arg \left(\mathbf{x}-\mathbf{z}_{i}\right)\right)
\end{aligned}
$$

is well defined for $\mathbf{x} \in D_{\mathbf{z}_{i}}$. With the change of indexes $p_{1}=m-l$ and $q_{1}=l$, we have

$$
\begin{aligned}
& \Psi(\mathbf{x})=\sum_{p_{1} \in \mathbb{Z}} \alpha_{p_{1}} {\left[\sum_{q_{1} \in \mathbb{Z}} H_{q_{1}}^{(1)}\left(k \mathbf{z}_{i}-\mathbf{z}_{j} \mid\right) J_{p_{1}+q_{1}}\left(k\left|\mathbf{x}-\mathbf{z}_{i}\right|\right)\right.} \\
&\left.\cdot \exp \left(-\imath q_{1}\left(\arg \left(\mathbf{z}_{i}-\mathbf{z}_{j}\right)-\pi\right)\right) \exp \left(\imath\left(p_{1}+q_{1}\right) \arg \left(\mathbf{x}-\mathbf{z}_{i}\right)\right)\right] .
\end{aligned}
$$

On the other hand, let us use formula (46) with $u=k\left|\mathbf{z}_{i}-\mathbf{z}_{j}\right|, v=k\left|\mathbf{x}-\mathbf{z}_{i}\right|$ and $w=k\left|\mathbf{x}-\mathbf{z}_{j}\right|$. The condition $|v|<|u|$ holds since $\mathbf{x}$ lies inside $D_{\mathbf{z}_{i}}$ whereas $\mathbf{z}_{j}$ lies outside $D_{\mathbf{z}_{i}}$ (because $D_{\mathbf{z}_{i}} \cap D_{\mathbf{z}_{j}}=\emptyset$ ). By Figure 5 , the two angles $\alpha$ and $\beta$ arising in formula (46) and Figure 4 satisfy $\alpha=\arg \left(\mathbf{z}_{i}-\mathbf{z}_{j}\right)+\pi-\arg \left(\mathbf{x}-\mathbf{z}_{i}\right)$ and $\beta=\arg \left(\mathbf{x}-\mathbf{z}_{j}\right)-\arg \left(\mathbf{z}_{i}-\mathbf{z}_{j}\right)$.

Hence by Graf's formula (46)

$$
\begin{aligned}
& H_{p_{1}}^{(1)}\left(k\left|\mathbf{x}-\mathbf{z}_{j}\right|\right) \exp \left(\imath p_{1}\left(\arg \left(\mathbf{x}-\mathbf{z}_{j}\right)-\arg \left(\mathbf{z}_{i}-\mathbf{z}_{j}\right)\right)\right) \\
& \quad=\sum_{q_{1} \in \mathbb{Z}} H_{p_{1}+q_{1}}^{(1)}\left(k\left|\mathbf{z}_{i}-\mathbf{z}_{j}\right|\right) J_{q_{1}}\left(k\left|\mathbf{x}-\mathbf{z}_{i}\right|\right) \exp \left(\imath q_{1}\left(\arg \left(\mathbf{z}_{i}-\mathbf{z}_{j}\right)+\pi-\arg \left(\mathbf{x}-\mathbf{z}_{i}\right)\right)\right) .
\end{aligned}
$$




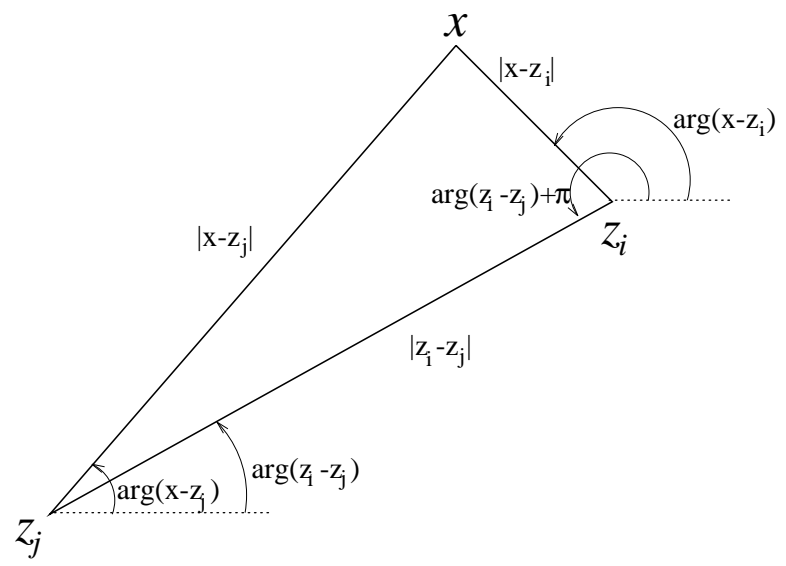

FIgURE 5. Link between $\widehat{u}, \widehat{v}, \widehat{w}$ and $\alpha, \beta$.

With the new index $q_{2}=-p_{1}-q_{1}$, and thanks to (35), we arrange the above formula as

$$
\begin{gathered}
H_{p_{1}}^{(1)}\left(k\left|\mathbf{x}-\mathbf{z}_{j}\right|\right) \exp \left(\imath p_{1}\left(\arg \left(\mathbf{x}-\mathbf{z}_{j}\right)-\arg \left(\mathbf{z}_{i}-\mathbf{z}_{j}\right)\right)\right) \\
=\sum_{q_{2} \in \mathbb{Z}} H_{-q_{2}}^{(1)}\left(k\left|\mathbf{z}_{i}-\mathbf{z}_{j}\right|\right) J_{-p_{1}-q_{2}}\left(k\left|\mathbf{x}-\mathbf{z}_{i}\right|\right) \\
\cdot \exp \left(-\imath\left(p_{1}+q_{2}\right)\left(\arg \left(\mathbf{z}_{i}-\mathbf{z}_{j}\right)-\pi-\arg \left(\mathbf{x}-\mathbf{z}_{i}\right)\right)\right) \\
=\sum_{q_{2} \in \mathbb{Z}} H_{q_{2}}^{(1)}\left(k\left|\mathbf{z}_{i}-\mathbf{z}_{j}\right|\right) J_{p_{1}+q_{2}}\left(k\left|\mathbf{x}-\mathbf{z}_{i}\right|\right) \exp \left(\imath q_{2}\left(\pi-\arg \left(\mathbf{z}_{i}-\mathbf{z}_{j}\right)+\arg \left(\mathbf{x}-\mathbf{z}_{i}\right)\right)\right) \\
\cdot \exp \left(\imath p_{1}\left(\arg \left(\mathbf{x}-\mathbf{z}_{i}\right)-\arg \left(\mathbf{z}_{i}-\mathbf{z}_{j}\right)\right)\right) .
\end{gathered}
$$

Then

$$
\begin{aligned}
H_{p_{1}}^{(1)}\left(k\left|\mathbf{x}-\mathbf{z}_{j}\right|\right) \exp \left(\imath p_{1} \arg \left(\mathbf{x}-\mathbf{z}_{j}\right)\right)=\sum_{q_{2} \in \mathbb{Z}} H_{q_{2}}^{(1)}\left(k\left|\mathbf{z}_{i}-\mathbf{z}_{j}\right|\right) J_{p_{1}+q_{2}}\left(k\left|\mathbf{x}-\mathbf{z}_{i}\right|\right) \\
\cdot \exp \left(-\imath q_{2}\left(\arg \left(\mathbf{z}_{i}-\mathbf{z}_{j}\right)-\pi\right)\right) \exp \left(\imath\left(p_{1}+q_{2}\right) \arg \left(\mathbf{x}-\mathbf{z}_{i}\right)\right) .
\end{aligned}
$$

By putting this into the expression for $\Psi$, we obtain

$$
\Psi(\mathbf{x})=\sum_{m \in \mathbb{Z}} \alpha_{m} H_{m}^{(1)}\left(k\left|\mathbf{x}-\mathbf{z}_{j}\right|\right) \exp \left(\imath m \arg \left(\mathbf{x}-\mathbf{z}_{j}\right)\right) \equiv \Phi(\mathbf{x}) .
$$

Proposition 10. Let $\Phi$ be a function defined in $D_{\mathbf{z}_{i}}$ and having the following expansion

$$
\Phi(\mathbf{x})=\sum_{m \in \mathbb{Z}} \alpha_{m} J_{m}\left(k\left|\mathbf{x}-\mathbf{z}_{i}\right|\right) \exp \left(\imath m \arg \left(\mathbf{x}-\mathbf{z}_{i}\right)\right)
$$


and moreover assume that the expansion $\sum_{m \in \mathbb{Z}} \alpha_{m} \exp (\imath m \theta) \exp (-\imath m \pi / 2)$ converges absolutely for all $\theta \in[0,2 \pi]$. Then for $\mathbf{x} \in D_{\mathbf{z}_{i}}$

$$
\begin{aligned}
\Phi(\mathbf{x})=\frac{1}{2 \pi} \int_{0}^{2 \pi} \exp \left(\imath k\left|\mathbf{x}-\mathbf{z}_{i}\right| \cos \left(\theta-\arg \left(\mathbf{x}-\mathbf{z}_{i}\right)\right)\right) \\
\cdot\left(\sum_{m \in \mathbb{Z}} \alpha_{m} \exp (\imath m \theta) \exp (-\imath m \pi / 2)\right) d \theta .
\end{aligned}
$$

Proof. The integral formula (44) enables us to write

$$
\begin{gathered}
\Phi(\mathbf{x})=\sum_{m \in \mathbb{Z}} \frac{\alpha_{m} \exp \left(\imath m \arg \left(\mathbf{x}-\mathbf{z}_{i}\right)\right)}{2 \pi} \\
\cdot \int_{0}^{2 \pi} \exp \left(\imath k\left|\mathbf{x}-\mathbf{z}_{i}\right| \cos \vartheta\right) \exp (\imath m \vartheta) \exp (-\imath m \pi / 2) d \vartheta .
\end{gathered}
$$

Under the assumption that the expansion $\sum_{m \in \mathbb{Z}} \alpha_{m} \exp (\imath m \vartheta) \exp (-\imath m \pi / 2)$ converges absolutely, we can perform the interchange of summation and integration to arrive at

$$
\begin{aligned}
\Phi(\mathbf{x})=\frac{1}{2 \pi} \int_{0}^{2 \pi} \exp \left(\imath k\left|\mathbf{x}-\mathbf{z}_{i}\right| \cos \vartheta\right) \\
\cdot\left(\sum_{m \in \mathbb{Z}} \alpha_{m} \exp \left(\imath m \arg \left(\mathbf{x}-\mathbf{z}_{i}\right)\right) \exp (\imath m \vartheta) \exp (-\imath m \pi / 2)\right) d \vartheta
\end{aligned}
$$

To conclude the proof, it only remains to do the change of variable $\theta=\vartheta+\arg \left(\mathbf{x}-\mathbf{z}_{i}\right)$.

\section{Appendix C: Some analytic Results.}

We give here some results that are very useful in Sections 4 and 5 .

Proposition 11. (i) For any $m \geq 0$ and any $l>m$

$$
\frac{l^{l}}{(l-m)^{l-m}} \leq l^{m} \mathrm{e}^{m} \text {. }
$$

(ii) For any $m \geq 0$ and any $l>0$

$$
\frac{l^{l}}{(l+m)^{l+m}} \leq l^{-m} \mathrm{e}^{-m}
$$

(iii) For any $m \geq 0$ and any $l \geq 1$

$$
\frac{(l+m)^{l+m}}{l^{l}} \leq l^{m}(m+1)^{m+1}
$$

Proof. $\quad$ (i) One may write

$$
\log \left(\frac{l^{l}}{(l-m)^{l-m}}\right)=m \log l-(l-m) \log \left(1-\frac{m}{l}\right) .
$$

Let us define $h(l)=-(l-m) \log \left(1-\frac{m}{l}\right)$. For $l>m \geq 0, h$ is a positive and increasing function of $l$, since

$$
h^{\prime}(l)=-\log \left(1-\frac{m}{l}\right)-\frac{l-m}{1-\frac{m}{l}} \frac{m}{l^{2}}=-\log \left(1-\frac{m}{l}\right)-\frac{m}{l} \geq 0 .
$$


Here we have used the fact that $-\log (1-u) \geq u$ for $0 \leq u<1$. Hence $0 \leq h(l) \leq \lim _{l \rightarrow \infty} h(l)=m$. Consequently $(i)$ isproved.

(ii) If we now define $h(l)=(l+m) \log \left(1+\frac{m}{l}\right)$, then we have

$$
\log \left(\frac{l^{l}}{(l+m)^{l+m}}\right)=-m \log l-h(l) .
$$

For $m \geq 0$ and $l>0, h$ is a positive function and

$$
h^{\prime}(l)=\log \left(1+\frac{m}{l}\right)-\frac{l+m}{1+\frac{m}{l}} \frac{m}{l^{2}}=\log \left(1+\frac{m}{l}\right)-\frac{m}{l} \leq 0 .
$$

Hence $h(l) \geq \lim _{l \rightarrow \infty} h(l)=m$, so that (ii) is proved.

(iii) With the previous definition of $h(l), h$ can be bounded by

$$
\log \left(\frac{(l+m)^{l+m}}{l^{l}}\right)=m \log l+h(l) .
$$

Since the function $h(l)$ is decreasing and $l \geq 1$, we get

$$
h(l) \leq h(1)=(1+m) \log (1+m) .
$$

This concludes the proof.

Proposition 12. Let $a$ and $A$ be two positive numbers. Then

(i) If $x \geq y \geq a$, then $\left(\frac{a}{x}\right)^{x} \leq\left(\frac{a}{y}\right)^{y}$.

(ii) If $y \geq x \geq a$, then $\left(\frac{x}{a}\right)^{x} \leq\left(\frac{y}{a}\right)^{y}$.

(iii) If $A<\frac{a}{\mathrm{e}}$, then $\max _{0 \leq x \leq A}\left(\frac{a}{x}\right)^{x}=\left(\frac{a}{A}\right)^{A}$.

(iv) If $A \geq \frac{a}{\mathrm{e}}$, then $\max _{0 \leq x \leq A}\left(\frac{a}{x}\right)^{x}=\exp \left(\frac{a}{\mathrm{e}}\right) \leq \exp (A)$.

Proof. As far as the first point (i) is concerned, since $\frac{a}{x} \leq \frac{a}{y} \leq 1$, we have directly

$$
\left(\frac{a}{x}\right)^{x}=\left(\frac{a}{x}\right)^{y}\left(\frac{a}{x}\right)^{x-y} \leq\left(\frac{a}{x}\right)^{y} \leq\left(\frac{a}{y}\right)^{y} .
$$

The second point can be derived in the same way.

Let $f(x)=\left(\frac{a}{x}\right)^{x}$. Its derivative is $f^{\prime}(x)=\left(\frac{a}{x}\right)^{x} \log \left(\frac{a}{\mathrm{e} x}\right)$. Hence, $f$ is an increasing function for $0 \leq x \leq \frac{a}{\mathrm{e}}$, and is a decreasing function for $x \geq \frac{a}{\mathrm{e}}$. The two points (iii), (iv) follow directly from this. We also notice that the maximum of $f$ is $f\left(\frac{a}{\mathrm{e}}\right)=\exp \left(\frac{a}{\mathrm{e}}\right)$.

Proposition 13. Let $x$ be a real number satisfying $0 \leq x<\frac{1}{\sqrt{\mathrm{e}}}$. Then for all integers $L$ and $M$, with $L \geq M$, we have

$$
\sum_{l=L}^{\infty} l^{M} x^{l} \leq C_{g} \frac{(2 L)^{M} x^{L}}{(1-x)^{M+1}}
$$

where $C_{g}:=\frac{1}{1-\sqrt{\mathrm{e}} x}$.

Proof. Let us introduce, for $L \geq M$, the two functions $f(x)=\sum_{l=L}^{\infty} l^{M} x^{l}$ and $g(x)=\sum_{l=L}^{\infty} x^{l}$ which are linked by the relation

$$
\left(x \frac{d}{d x}\right)^{M} g(x)=f(x)
$$


Moreover, $g(x)=\frac{x^{L}}{1-x}$. From this relation for $g$, one can easily see that there exists $M+1$ coefficients $P_{0}^{M}, \ldots, P_{M}^{M}$ such that

$$
\left(x \frac{d}{d x}\right)^{M} g(x)=\frac{P_{0}^{M} x^{L}+P_{1}^{M} x^{L+1}+\cdots+P_{M}^{M} x^{L+M}}{(1-x)^{M+1}} .
$$

A straightforward calculation shows the recursive relations

$$
\left\{\begin{array}{l}
P_{0}^{M+1}=L P_{0}^{M}, \\
P_{m}^{M+1}=(L+m) P_{m}^{M}+(M+2-L-m) P_{m-1}^{M}, 1 \leq m \leq M, \\
P_{M+1}^{M+1}=(1-L) P_{M}^{M}
\end{array}\right.
$$

with $P_{0}^{0}=1$. We claim that

$$
\left|P_{m}^{M}\right| \leq(2 L+m)^{M}, 0 \leq m \leq M .
$$

The proof of this inequality is recursive. First we notice that (47) is obvious when $M=0$. Then we assume that (47) is satisfied at the level $M$. For $1 \leq m \leq M$, we have

$$
\begin{aligned}
\left|P_{m}^{M+1}\right| & \leq(L+m)(2 L+m)^{M}+(L+m-M-2)(2 L+m-1)^{M} \\
& \leq(L+m)(2 L+m)^{M}+L(2 L+m)^{M}=(2 L+m)^{M+1} .
\end{aligned}
$$

When $m=M+1,\left|P_{M+1}^{M+1}\right| \leq(L-1)(2 L+M)^{M} \leq(2 L+M+1)^{M+1}$. And finally, when $m=0$, we have $\left|P_{0}^{M+1}\right| \leq L(2 L)^{M} \leq(2 L)^{M+1}$. Hence (47) holds for all $M \in \mathbb{N}$. Now the function $f$ can be bounded by

$$
f(x) \leq \frac{\sum_{m=0}^{M}\left|P_{m}^{M}\right| x^{L+m}}{(1-x)^{M+1}} \leq \frac{\sum_{m=0}^{M}(2 L+m)^{M} x^{L+m}}{(1-x)^{M+1}} .
$$

From the relation (which holds since $L \geq M$ )

$$
\log \left[\frac{(2 L+m)^{M}}{(2 L)^{M}}\right]=M \log \left(1+\frac{m}{2 L}\right) \leq M \frac{m}{2 L} \leq \frac{m}{2},
$$

and since the exponential is an increasing function, we obtain

$$
(2 L+m)^{M} \leq(2 L)^{M} \exp \left(\frac{m}{2}\right) .
$$

Hence

$$
f(x) \leq(2 L)^{M} x^{L} \frac{\sum_{m=0}^{M}(\sqrt{\mathrm{e}} x)^{m}}{(1-x)^{M+1}} .
$$

Since $x<\frac{1}{\sqrt{\mathrm{e}}}, \sum_{m=0}^{M}(\sqrt{\mathrm{e}} x)^{m} \leq C_{g}:=\frac{1}{1-\sqrt{\mathrm{e}} x}$. Hence the proposition is proved.

Proposition 14. The sequence $S_{m}:=\frac{1}{N_{T}} \sum_{n_{T}=1}^{N_{T}} \exp \left(\imath m \frac{2 \pi n_{T}}{N_{T}}\right)$ satisfies for $m \in$ $\mathbb{Z}$

$$
S_{0}=1, \quad S_{m}=0 \quad \text { for } 1 \leq|m| \leq N_{T}-1
$$


Proof. The first equality is obvious. The complex numbers $x_{n_{T}}:=\exp \left(\frac{2 \imath \pi n_{T}}{N_{T}}\right)$ are the zeros of the polynomial

$$
P(x)=x^{N_{T}}-1=\sum_{m=0}^{N_{T}} p_{m} x^{N_{T}-m},
$$

where $p_{0}=1, p_{N_{T}}=-1$, and $p_{m}=0$ for $m \in\left\{1, \cdots, N_{T}-1\right\}$. The moments of $x_{n_{T}}$, defined by

$$
\sigma_{m}:=\sum_{n_{T}=1}^{N_{T}}\left(x_{n_{T}}\right)^{m}=N_{T} S_{m},
$$

are linked to the coefficients $p_{m}$ by the Newton formula

$$
-m p_{m}=\sum_{l=0}^{m-1} p_{l} \sigma_{m-l}=\sigma_{m} .
$$

We conclude that $\sigma_{m}=0$ and hence $S_{m}=0$ for $1 \leq m \leq N_{T}-1$. The previous analysis can still be done with the sequence $y_{n_{T}}=\exp \left(-\frac{2 \imath \pi n_{T}}{N_{T}}\right)$ instead of $x_{n_{T}}$. We remark that a positive integer $m$ with $y_{n_{T}}$ corresponds to $-m$ with $x_{n_{T}}$. Thus, the previous analysis is still valid for negative values of $m$.

\section{ACKNOWLEDGMENT}

I would like to thank E. Darve for some fruitful remarks on the paper. I am also grateful to P. Monk for helpful discussions and encouragement.

\section{REFERENCES}

1. M. Abramowitch, I.A. Stegun, Handbook of Mathematical Functions with Formulas, Graphs and Mathematical Tables, Wiley, New York, 1964. MR 29:4914

2. F. Canning, The Impedance Matrix Localization Method for Moment-Method Calculations, IEEE antenna propag., Vol 32, pp 18-30, oct 1990.

3. F. Canning, Improved Matrix Localization, IEEE antenna propag., Vol 41,No 5, pp 659-667, may 1993.

4. R. Coifman, G. Beylkin, V. Rokhlin, Fast Wavelet Transforms and Numerical Algorithms, Comm. Pure Appl. Math., 44, pp 141-183, 1991. MR 92c:65061

5. D. Colton, R. Kress, Integral Equation Methods in Scattering Methods in Scattering Theory, Wiley, 1983. MR 85d:35001

6. L. Greengard, V. Rokhlin, A Fast Algorithm for Particle Simulations, J. Comput. Phys., Vol 73, pp 325-348, 1987. MR 88k:82007

7. M.A. Hamdi, Une Formulation Variationnelle par des Équations Intégrales pour la Résolution de l'Équation de Helmholtz avec des Conditions aux Limites Mixtes, C. R. Acad. Sci. Paris, 292, série II, pp 17-20, 1981. MR 82k:35027

8. A. Harten, I. Yad-Shalom, Fast Multiresolution Algorithms for Matrix-Vector Multiplication, SIAM J. Numer. Anal., Vol 31, No 4, pp 1191-1218, Aug 1994. MR 95f:65223

9. A. Harten, Discrete Multi-Resolution Analysis and Generalized Wavelets, Appl. Numer. Math., Vol 12, pp 153-192, 1993. MR 95b:65163

10. J. Jin, The Finite Element Method in Electromagnetics, Wiley interscience, New York, 1993.

11. P. Martin, Multipole Scattering: an Invitation, in The third international conference on mathematical and numerical aspects of wave propagation, G. Cohen, 1995.

12. H. Petersen, D. Soelvason, J. Perran, E. Smith, Error Estimates for the Fast Multipole Method. I. The Two-Dimensional Case, Proceedings of the Royal Society of London, serie A, Vol 448, pp 389-400, march 1995.

13. V. Rokhlin, Rapid Solution of the Integral Equations of Classical Potential Theory, J. Comput. Phys., 60, pp 187-207, 1985. MR 86k:65120 
14. V. Rokhlin, Rapid Solution of Integral Equations of Scattering Theory in Two Dimensions, J. Comput. Phys., 86, pp 414-439, 1990. MR 90k:76081

15. V. Rokhlin, B. Alpert, A Fast Algorithm for the Evaluation of Legendre Expansions, SIAM J. Sci. Stat. Comput., Vol 12, No 1, pp 158-179, Jan 1991. MR 91i:65042

16. V. Rokhlin, N. Engheta, W. Murphy, M. Vassiliu, The Fast Multipole Method for Electromagnetic Scattering Problems, IEEE transactions on antenna and propagation, Vol 40, No 6, pp 634-641, june 1992. CMP 92:14

17. V. Rokhlin, R. Coifman, S. Wandzura The Fast Multipole Method for Wave Equation: a Pedestrian Prescription, IEEE anten. and propag. mag., Vol 35, No 3, pp 7-12, june 1993.

18. V. Rokhlin Diagonal Forms of Translation Operators for the Helmholtz Equation in Three Dimensions, Appl. and Comput. Harmonic Analysis, Vol 1, pp 82-93, 1993. MR 95d:35034

Thomson CSF-LCR, Domaine de Corbeville, 91404 Orsay Cedex, France

E-mail address: labreuch@thomson-lcr.fr 